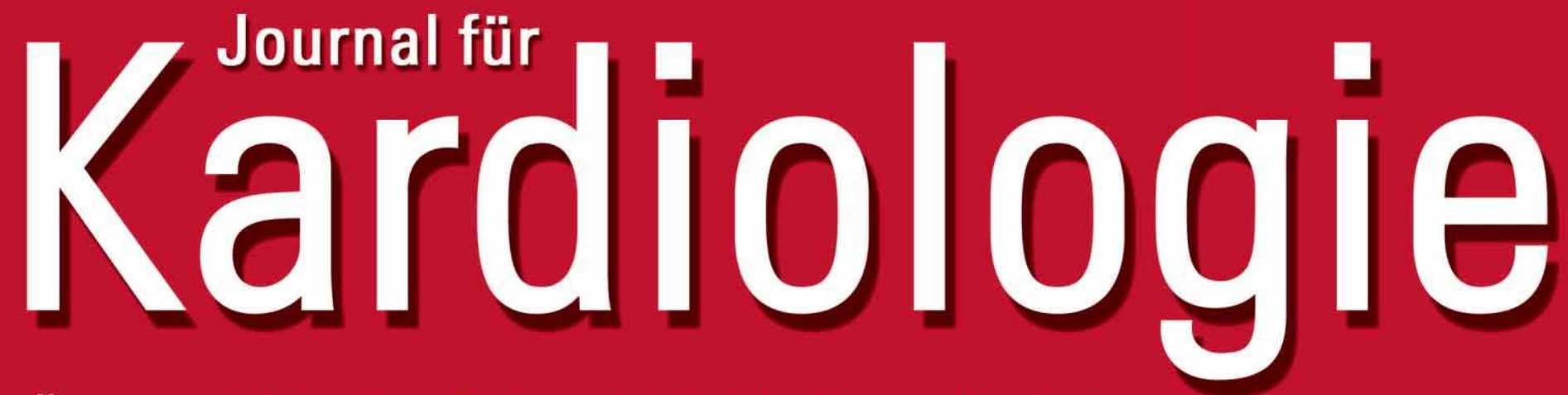

Österreichische Zeitschrift für Herz-Kreislauferkrankungen

\title{
Praxisleitlinien Ergometrie
}

Wonisch M, Berent R, Klicpera M, Laimer H Marko C, Schwann H, Schmid P Journal für Kardiologie 2008; 15 (Supplementum A - Praxisleitlinien Ergometrie), 3-17

\section{Homepage:}

\section{www.kup.at/kardiologje}

\author{
Online-Datenbank mit \\ Autoren- und Stichwortsuche
}

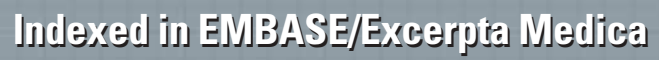

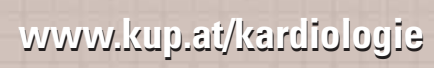

Krause \& Pachernegg GmbH · VERLAG für MEDIZIN und WIRTSCHAFT · A-3003 Gablitz 


\section{Praxisleitlinien Ergometrie *}

M. Wonisch, R. Berent, M. Klicpera, H. Laimer, C. Marko, R. Pokan, P. Schmid, H. Schwann

für die AG Kardiologische Rehabilitation und Sekundärprävention der Österreichischen Kardiologischen Gesellschaft

Kurzfassung: Die Ergometrie stellt ein Standardverfahren in der Kardiologie dar. Dies hat sich auch durch neue diagnostische Verfahren nicht wesentlich geändert. Ziel dieser Leitlinien ist es, eine praxisrelevante Übersicht über die Ergometrie zu geben. Neben den physiologischen und leistungs diagnostischen Grundlagen wird eine Übersicht übe Vorraussetzungen, Indikationen, Kontraindikationen und Abbruchkriterien gegeben. Auch werden Empfehlungen für die Durchführung, Bewertung der erhobenen Parameter und verschiedene Einflussgrößen angeführt.

Abstract: Practice Guidelines for Exercise Testing. Exercise testing is a standard procedure in cardiology. This has not changed with newer diag- nostic procedures. The purpose of this recommenda tion is to give a practical overview about ergometry. It contains physiologic fundamentals as well as an overview about assumptions, indications, contraindications and criteria about interruption. Recommendations about the procedure, valuation of measured parameters and different influences are given. J Kardiol 2008; 15 (Suppl A): 3-17.

\section{Einleitung}

Belastungsuntersuchungen spielen in der Kardiologie seit Anbeginn eine große Rolle [1-3]. Dies hat sich auch durch neue diagnostische Verfahren nicht wesentlich geändert [4-6].

Ziel der vorliegenden Empfehlungen ist es, eine aktuelle Übersicht über die praktische Anwendung der klassischen Ergometrie zu geben.

\section{Physiologische und pathophysiologi- sche Grundlagen}

Dynamische Belastungen führen zu einer Steigerung des Herz-Zeit-Volumens (Schlagvolumen- und Herzfrequenzanstieg) und des myokardialen Sauerstoffverbrauchs. Einen indirekten Ausdruck dieses myokardialen Sauerstoffbedarfs stellt das „Druck-Frequenz-Produkt (DFP)“ dar, welches aus dem Produkt von systolischem Blutdruck und Herzfrequenz gebildet wird.

Unter ischämischen Bedingungen kommt die sog. „Ischämiekaskade" zum Tragen, die besagt, dass sich ischämische Veränderungen im Myokard zuerst durch Stoffwechselveränderungen, dann durch Änderungen der myokardialen Funktion gefolgt von EKG-Veränderungen und zuletzt durch klinische Beschwerden manifestieren [7] (Abb. 1).

Während stufenförmig ansteigender Körperarbeit kann die Energiebereitstellung in 3 Phasen unterteilt werden [8-12] (Abb. 2).

- Phase I - die aerobe Phase bis zum ersten Laktatanstieg und bis zum Beginn einer überproportionalen Zunahme der Ventilation gegenüber der Sauerstoffaufnahme ohne zusätzliche Steigerung der Kohlendioxydabgabe.

- Phase II - die aerob-anaerobe Übergangsphase, die Phase der respiratorischen Kompensation der metabolischen Azidose, bis zum zweiten Laktatanstieg und bis zu einer nun

\footnotetext{
* Vorstandsbeschluss der Österreichischen Kardiologischen Gesellschaft vom 28.05.2008
}

Korrespondenzadresse: Prim. Doz. DDr. Manfred Wonisch, SonderkrankenanstaltRehabilitationszentrum für Herz-Kreislauferkrankungen, A-8061 St. Radegund, Quellenstraße 1; E-Mail: manfred.wonisch@pva.sozvers.at einsetzenden überschießenden Steigerung der Ventilation bezogen auf die Kohlendioxydabgabe.

- Phase III - die anaerobe Phase bis zur Ausbelastung [9] (Abb. 2).

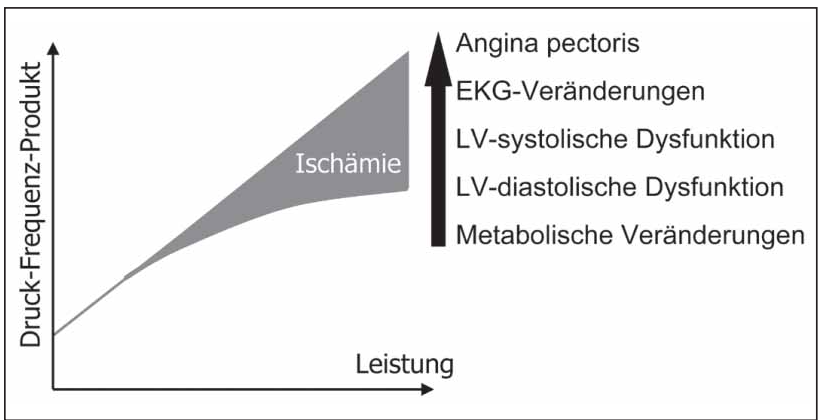

Abbildung 1: Ischämiekaskade

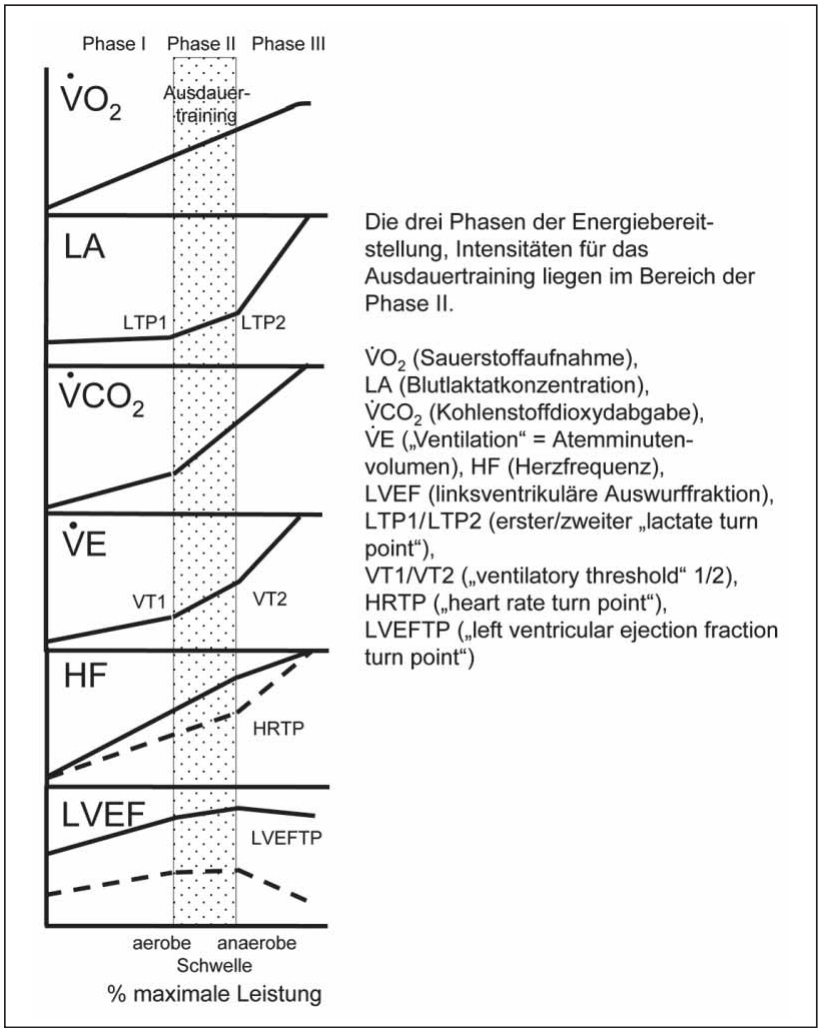

Abbildung 2: Phasenkonzept 
Im Gegensatz zu Gesunden, wo ein deutlicher Anstieg der linksventrikulären Auswurffraktion (LVEF) im Sinne einer Zunahme der myokardialen Funktion von Ruhe bis zum Übergang von Phase II zu Phase III mit einer anschließenden Plateau-Bildung oder einer geringfügigen Abnahme der LVEF zu beobachten ist $[8,13]$, kommt es bei Patienten mit koronarer Herzerkrankung zu einer deutlichen Abnahme der LVEF während Phase III der Energiebereitstellung. Als Zeichen der myokardialen Überlastung kann die LVEF bei maximaler Belastung auf Werte unter den Ruhewert bei gleichzeitiger Zunahme des enddiastolischen Volumens absinken [8, 14].

Auch der Herzfrequenzverlauf zeigt bei Gesunden unter ansteigender Belastung ein dazu passendes Verhalten mit einer Abflachung in der Phase III der Energiebereitstellung, welche mit der Plateaubildung der LVEF einhergeht $[13,15]$. Mit zunehmendem Alter lässt diese Abflachung allerdings in Abhängigkeit zur myokardialen Funktion nach. Demgegenüber findet man bei den meisten Patienten mit koronarer Herzkrankheit und eingeschränkter linksventrikulärer Funktion eine weitere Zunahme der Anstiegsgeschwindigkeit der Belastungsherzfrequenz $[14,16]$.

\section{Voraussetzungen zur Ergometrie}

\subsection{Aufklärung}

Eine vorangehende adäquate Patientenaufklärung (mündlich und schriftlich !!) mit entsprechender Dokumentation ist unbedingt erforderlich.

\subsection{Klinische Voraussetzungen}

- Exakte Zuweisung bzw. Fragestellung

- Anamnese, körperliche Untersuchung inklusive Auskultation, Ruhe-RR, Ruhe-EKG im Liegen

- Fakultativ: Echokardiographie, Thorax-Röntgen, Lungenfunktion

- Medikamentenanamnese (s. Einflussgrößen)

- Mindestens 2 Stunden Abstand zur letzten Mahlzeit und ausreichende Regeneration nach schweren körperlichen Belastungen

- Mindestens 12 Stunden Abstand nach Alkohol und 2 Stunden Abstand nach Nikotin

\subsection{Räumliche Voraussetzungen}

Angenehmes Raumklima mit Raumtemperatur $18-24^{\circ} \mathrm{C}$, relativer Luftfeuchte 30-60 \%; ausreichende Raumgröße mit Liege in unmittelbarer Nähe.

\subsection{Apparative Voraussetzungen}

\subsubsection{Ergometer}

- Anforderungen: standardisierbar, reproduzierbar, dosierbar

- Fahrrad- und Laufbandergometrie erfüllen diese Kriterien am besten

- Fahrradergometer: vorzugsweise drehzahlunabhängig elektrisch gebremst

- Bei speziellen Gegebenheiten und Erfordernissen: alternativ Armkurbel, sportartspezifische Ergometer (z. B. Rollstuhlergometrie, Ruderergometer)

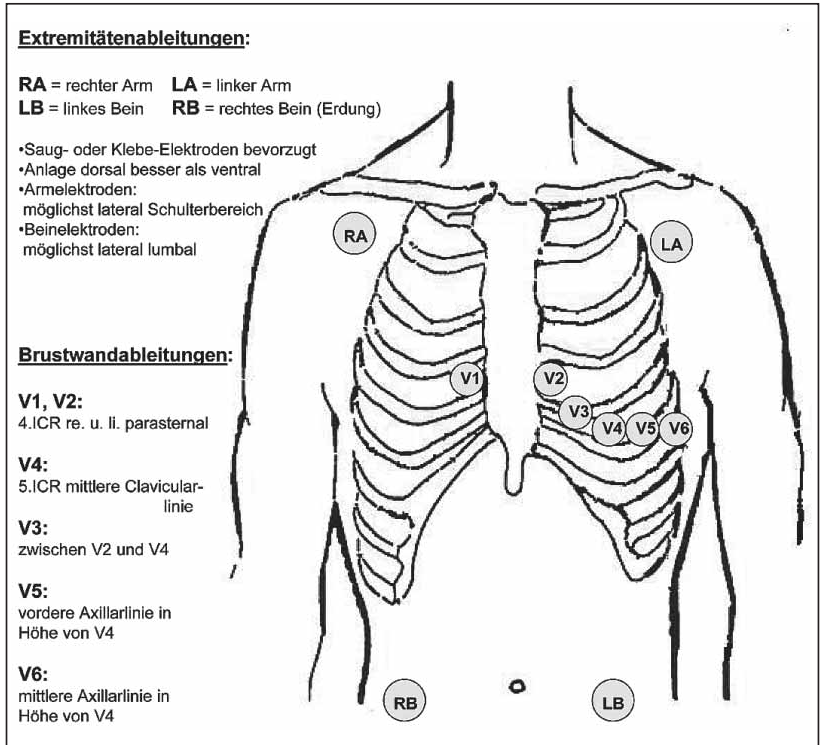

Abbildung 3: Elektrodenanlage. Mod. nach [5].

- CE-Zeichen, Wartung und Eichung nach Herstellerempfehlung

\subsubsection{EKG-Registrierung}

- 12-Kanal-Registrierung (Extremitäten- und Brustwandableitungen) (Abb. 3)

- Bevorzugt Rechner-gestützte EKG-Geräte mit Signalmittelung und einstellbarer Markierung der Messpunkte in der EKG-Kurve, Vorprogrammierung des Untersuchungsablaufs

- Nicht-Rechner-gestütztes EKG: Nullpunktstabilisierung, Ausschriebgeschwindigkeit 25 oder $50 \mathrm{~mm} / \mathrm{sec}$, fortlaufende Registrierung mit langsamem Papiervorschub

- EKG-Ausschrieb alle 2 Minuten, bei Testabbruch, 1, 3 und 5 Min. nach Belastung, Weiter-Registrierung nach Bedarf bis Erreichen des Ausgangs-EKGs und klinischer Stabilisierung des Probanden

- Gewährleistung einer guten Signalqualität durch entsprechende Hautpräparation (ev. Rasur)

- Kontinuierliche Monitorüberwachung (mindestens 2 Ableitungen)

- CE-Zeichen, Gerätewartung entsprechend Herstellerempfehlung

\subsubsection{Notfallsausrüstung}

- Stethoskop, RR-Messgerät

- Liege in unmittelbarer Nähe

- Defibrillator: CE-Zeichen, Überprüfung zu Beginn jedes Arbeitstages, Gerät während Ergometrie eingeschaltet, Wartung nach Herstellerempfehlung

- Intubationsbesteck, Tubus, Ambubeutel, $\mathrm{O}_{2}$-Flasche

- Spritzen, Nadeln, Verweilkanülen, Stauschlauch, Desinfektionsmittel

- Telefon im Raum!!!

- Medikamente: Adenosin, Adrenalin, Ajmalin, Amiodaron, Atropin, Betablocker, Diazepam, Furosemid, Lidocain, Theophyllin, Verapamil;

Infusionslösung $(0,9 \% \mathrm{NaCl}$ oder $5 \%$ Glukose $)$ mit Infusionsbesteck;

Nitroglyzerin, Fenoterol DA, Dextrose oral 


\subsection{Personelle Voraussetzungen}

\subsubsection{Qualifizierte/r Arzt/Ärztin}

- FachärztIn für Innere Medizin, vorzugsweise mit Additivfach Kardiologie oder Internistische Sportheilkunde

- Ärzte/Ärztinnen mit jus practicandi, die im Rahmen der Ausbildung zumindest 100 Ergometrien nachgewiesen haben

- Nachweis von jährlich zumindest 50 durchgeführten Ergometrien

- Kenntnisse in (Patho)physiologie des Herz-Kreislauf-Systems, Leistungsphysiologie und Trainingsberatung

- Erfahrung in der Durchführung von Notfall- und Reanimationsmaßnahmen

- Ständige Verfügbarkeit im Untersuchungsraum

- Kontinuierliche Beobachtung des Probanden (Symptomatik, Hautkolorit, Kaltschweißigkeit, Erschöpfung)

\subsubsection{Qualifizierte Hilfskraft}

Ausgebildetes medizinisches Personal mit :

- Erfahrung in der Registrierung von Ruhe- und BelastungsEKG (Hautpräparation, Elektrodenplatzierung, Fehlerund Artefakt-Management)

- Ausreichender Geräteeinschulung

- Erfahrung in der Blutdruckmessung

- Kenntnissen in kardialer Beschwerdesymptomatik

- Basis-Kenntnissen in Ischämie- und Arrhythmiediagnostik
- Kenntnissen der wichtigsten Maßnahmen der Reanimation und Notfallmedizin

\section{Indikationen}

Die Indikationen zu Belastungsuntersuchungen werden in 3 Klassen eingeteilt [17]:

- Klasse I: Gesicherte Indikationen (generelle Akzeptanz)

- Klasse II: Mögliche Indikationen

- II a: Meinungen mit eher ,positivem Aspekt“ für Bedeutung und/oder Durchführung einer Belastungsuntersuchung

- II b: Meinungen mit eher ,negativem Aspekt“ für Bedeutung und/oder Durchführung einer Belastungsuntersuchung

- Klasse III: Keine Indikationen (generelle Ablehnung)

\subsection{Indikationen zur Belastungsuntersuchung zur Diagnostik und Verlaufskontrolle einer koro- naren Herzkrankheit}

Siehe Tabellen 1 und 2.

\subsection{Indikationen zur Belastungsuntersuchung nach Myokardinfarkt}

Siehe Tabelle 3.

Tabelle 1: Indikationen zur Belastungsuntersuchung zur Diagnostik und Verlaufskontrolle einer koronaren Herzkrankheit [4-6]

Klasse I Erwachsene Patienten mit mittlerem Risiko für eine KHK (auch Patienten mit komplettem Rechtsschenkelblock und ST-StreckenSenkung $<1 \mathrm{~mm}$ im Ruhe-EKG) nach Alter, Symptomen und Geschlecht (s. Tab. 2 - Vortestwahrscheinlichkeit)

Patienten mit klinischem Verdacht auf eine KHK oder bekannter KHK mit signifikanter Veränderung des klinischen Bildes

Klasse Ila Patienten mit bekannter KHK zur Verlaufskontrolle

Untersuchung von asymptomatischen Männern > 40 Jahre und Frauen > 50 Jahre vor mittelschweren bis schweren körperlichen Belastungen

Bei Berufen, bei denen eine Erkrankung Einfluss auf die öffentliche Sicherheit hat

Hohe Wahrscheinlichkeit für eine KHK (z. B. chronische Niereninsuffizienz, Diabetes mellitus)

Klasse Ilb Patienten mit hoher Wahrscheinlichkeit für eine KHK (nach Alter, Symptomen, Geschlecht)

Patienten mit niedriger Wahrscheinlichkeit für eine KHK (nach Alter, Symptomen, Geschlecht)

ST-Strecken-Senkung $<1 \mathrm{~mm}$ im Ruhe-EKG unter Digitalis-Medikation

linksventrikuläre Hypertrophiezeichen und ST-Strecken-Senkungen $<1 \mathrm{~mm}$ im Ruhe-EKG

Klasse III Abnorme Befunde im EKG (Präexzitationssyndrom, z. B. Wolff-Parkinson-White-Syndrom), permanente Schrittmacherstimulation, ST-Strecken-Senkung > $1 \mathrm{~mm}$ im Ruhe-EKG, kompletter Linksschenkelblock (QRS-Breite > 120 msek), frischer (akuter) Myokardinfarkt

schwere Begleiterkrankungen mit eingeschränkter Lebenserwartung

Patienten mit geplanter Revaskularisation

\begin{tabular}{|c|c|c|c|c|c|}
\hline Alter & Geschlecht & $\begin{array}{c}\text { Typische } \\
\text { Angina pectoris }\end{array}$ & $\begin{array}{c}\text { Atypische } \\
\text { Angina pectoris }\end{array}$ & $\begin{array}{l}\text { Unspezifischer } \\
\text { Thoraxschmerz }\end{array}$ & Asymptomatisch \\
\hline $30-39$ & $\begin{array}{l}\text { männlich } \\
\text { weiblich }\end{array}$ & $\begin{array}{l}\text { mittlere } \\
\text { mittlere }\end{array}$ & $\begin{array}{c}\text { mittlere } \\
\text { sehr niedrige }\end{array}$ & $\begin{array}{c}\text { niedrige } \\
\text { sehr niedrige }\end{array}$ & $\begin{array}{l}\text { sehr niedrige } \\
\text { sehr niedrige }\end{array}$ \\
\hline $40-49$ & $\begin{array}{l}\text { männlich } \\
\text { weiblich }\end{array}$ & $\begin{array}{l}\text { hohe } \\
\text { mittlere }\end{array}$ & $\begin{array}{l}\text { mittlere } \\
\text { niedrige }\end{array}$ & $\begin{array}{c}\text { mittlere } \\
\text { sehr niedrige }\end{array}$ & $\begin{array}{c}\text { niedrige } \\
\text { sehr niedrige }\end{array}$ \\
\hline $50-59$ & $\begin{array}{l}\text { männlich } \\
\text { weiblich }\end{array}$ & $\begin{array}{l}\text { hohe } \\
\text { mittlere }\end{array}$ & $\begin{array}{l}\text { mittlere } \\
\text { mittlere }\end{array}$ & $\begin{array}{l}\text { mittlere } \\
\text { niedrige }\end{array}$ & $\begin{array}{c}\text { niedrige } \\
\text { sehr niedrige }\end{array}$ \\
\hline $60-69$ & $\begin{array}{l}\text { männlich } \\
\text { weiblich }\end{array}$ & $\begin{array}{l}\text { hohe } \\
\text { hohe }\end{array}$ & $\begin{array}{l}\text { mittlere } \\
\text { mittlere }\end{array}$ & $\begin{array}{l}\text { mittlere } \\
\text { mittlere }\end{array}$ & $\begin{array}{l}\text { niedrige } \\
\text { niedrige }\end{array}$ \\
\hline
\end{tabular}




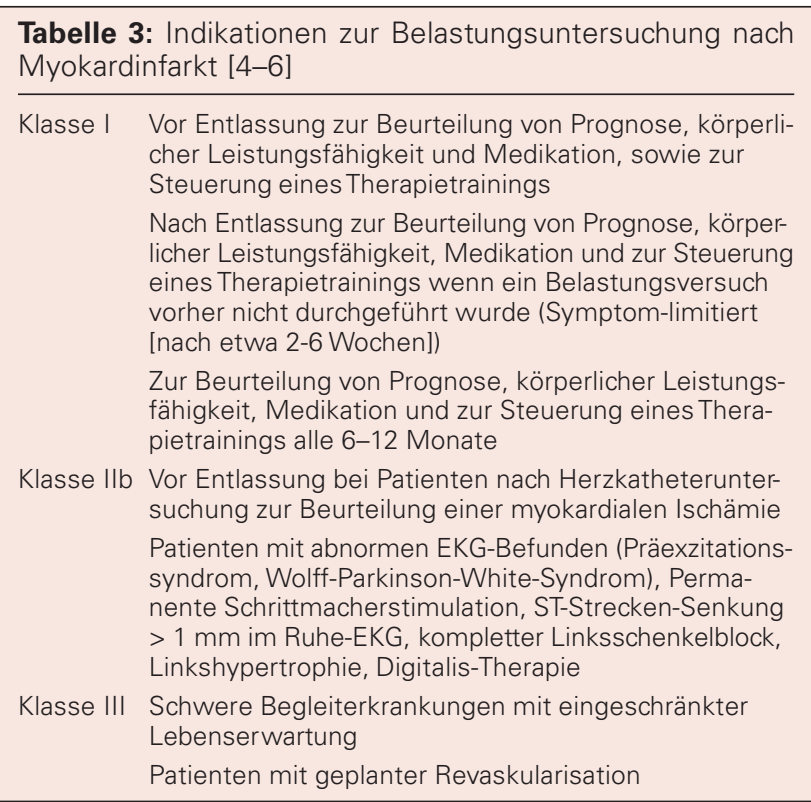

Tabelle 4: Indikationen zur Belastungsuntersuchung bei asymptomatischen Personen ohne bekannte koronare Herzkrankheit [4-6]

Klasse I Zur Beurteilung der körperlichen Leistungsfähigkeit sowie zur Steuerung eines Therapietrainings bei Patienten mit kardiovaskulären Risikofaktoren

Klasse Ila Zur Beurteilung der körperlichen Leistungsfähigkeit sowie zur Steuerung eines Ausdauertrainings bei allen körperlich aktiven Personen

Tabelle 5: Indikationen zur Belastungsuntersuchung vor und nach Revaskularisation [4-6]

Klasse I Nachweis myokardialer Ischämie vor etwaiger Revaskularisation

Beurteilung von Patienten mit rezidivierenden Symptomen (verdächtig für Ischämie) nach Revaskularisation Beratung über das Ausmaß einer körperlichen Aktivität und/oder des Trainingsprogramms einer kardialen Rehabilitation

Klasse lla Asymptomatische Patienten mit hohem Risiko einer Restenose 1 Monat nach Revaskularisation

Klasse Ilb Regelmäßige Beurteilung des Status bei asymptomatischen Patienten mit hohem Risiko für Restenose Bypass-Verschluss oder Progression der KHK

Klasse III Lokalisation der Ischämie zur Festlegung der Interventionsart

\subsection{Indikationen zur Belastungsuntersuchung} bei asymptomatischen Personen ohne bekannte koronare Herzkrankheit

Siehe Tabelle 4.

\subsection{Indikationen zur Belastungsuntersuchung vor und nach Revaskularisation}

Siehe Tabelle 5.

\subsection{Indikationen zur Belastungsuntersuchung bei Arrhythmiepatienten}

Siehe Tabelle 6.
Tabelle 6: Indikationen zur Belastungsuntersuchung bei Arrhythmie-Patienten [4-6, 18-20]

Klasse I Identifikation des optimalen Frequenzverhaltens bei frequenzadaptiven Schrittmacher-Systemen (Art der Belastung ist sensorabhängig)

Klasse Ila Patienten mit bekannten oder möglichen belastungsinduzierten Arrhythmien zur Diagnosestellung

Beurteilung des Herzfrequenzverhaltens unter Belastung bei Patienten mit Vorhofflimmern

Beurteilung therapeutischer Verfahren (medikamentös, Ablation, antitachykarde Chirurgie) bei Patienten mit belastungsinduzierten Arrhythmien

Beurteilung des Herzfrequenz- und Rhythmusverhaltens von Patienten mit ICD

Ausschluss proarrhythmischer Effekte nach Einleiten einer antiarrhythmischen Therapie

Beurteilung der chronotropen Kompetenz

Klasse Ilb Patienten mittleren Alters mit oder ohne KHK mit isolierten ventrikulären Extrasystolen

Asymptomatische junge Patienten mit isolierten Extrasystolen

Tabelle 7: Patienten mit arterieller Hypertonie [4-6, 21-23]

Klasse I Erkennung einer hypertensiven Belastungsreaktion bei normalem und hoch (noch) normalem Ruheblutdruck (s. Tab. 8 - Belastungsblutdruck)

Prognostische Aussagen (Entwicklung einer LVH, kardiovaskuläres Risiko bei Hypertonikern ohne Organmanifestation)

Wirksamkeit der antihypertensiven Therapie auf den Belastungsblutdruck

Einschätzung der Belastbarkeit und Trainingssteuerung unter antihypertensiver Therapie

Klasse Ila Diagnose bzw. Ausschluss einer Weißkittelhypertonie Pat. mit LVH unklarer Genese zur Beurteilung des RRVerhaltens

Klasse III Arterielle Hypertonie in Ruhe (RR $>180 / 100$ mmHg)

\begin{tabular}{|c|c|c|}
\hline Alter & $\begin{array}{c}75 \mathrm{Watt} \\
\mathrm{RR}(\mathrm{mmHg})\end{array}$ & $\begin{array}{c}100 \mathrm{Watt} \\
\mathrm{RR}(\mathrm{mmHg})\end{array}$ \\
\hline 20-50 Jahre & $185 / 100$ & $200 / 100$ \\
\hline 51-60 Jahre & $195 / 105$ & $210 / 105$ \\
\hline 61-70 Jahre & $205 / 110$ & $220 / 110$ \\
\hline
\end{tabular}

\subsection{Indikationen zur Belastungsuntersuchung von Patienten mit arterieller Hypertonie}

Siehe Tabellen 7 und 8.

\subsection{Indikationen zur Belastungsuntersuchung von Patienten mit Klappenvitien}

Siehe Tabelle 9.

\subsection{Indikationen zur Belastungsuntersuchung von Patienten mit kongenitalen Vitien}

Siehe Tabelle 10 


\begin{tabular}{ll}
\hline $\begin{array}{l}\text { Tabelle 9: Indikation zur Belastungsuntersuchung bei Pati- } \\
\text { enten mit Klappenvitien [4-6, 24, 25] }\end{array}$ \\
\hline Klasse I $\quad \begin{array}{l}\text { Asymptomatische hochgradige Aortenstenose zur } \\
\text { Demaskierung von klinischer Symptomatik, Beurtei- } \\
\text { lung des RR-Verhaltens und zur Risikostratifizierung }\end{array}$ \\
Klasse IIa $\begin{array}{l}\text { Zur Festlegung und Verlaufskontrolle der körperlichen } \\
\text { Leistungsfähigkeit und Objektivierung der klinischen } \\
\text { Symptomatik bei anderen Klappenvitien }\end{array}$ \\
Klasse IIb $\begin{array}{l}\text { Bei Patienten mit Mitralstenose zur Festlegung der } \\
\text { körperlichen Leistungsfähigkeit mit invasiver Messung } \\
\text { des pulmonalarteriellen Druckes }\end{array}$ \\
Klasse III $\quad$ Symptomatische hochgradige Aortenstenose
\end{tabular}

\begin{tabular}{l} 
Tabelle 10: Indikationen zur Belastungsuntersuchung bei \\
Patienten mit kongenitalen Vitien [26] \\
\hline Klasse Ila Zur Risikostratifizierung von Patienten mit HOCM \\
durch fehlenden oder abgeschwächten RR-Anstieg \\
Zur Festlegung und Verlaufskontrolle der körperlichen \\
Leistungsfähigkeit und Objektivierung der klinischen \\
Symptomatik bei anderen kongenitalen Vitien
\end{tabular}

\section{Kontraindikationen}

Um die Komplikationsrate bei Belastungsuntersuchungen möglichst gering zu halten, müssen vor einem Arbeitsversuch eventuelle Kontraindikationen unbedingt beachtet werden.

Sehr gut bewährt hat sich dabei die Einteilung in absolute und relative Kontraindikationen (Tab. 11).

Bei Vorliegen relativer Kontraindikationen ist eine Belastungsuntersuchung dann indiziert, wenn ein überwiegender Nutzen gegenüber dem Untersuchungsrisiko erwartet werden kann.

\section{Belastungsabbruch (Tab. 12)}

Ein Erreichen oder Überschreiten einer Sollleistung oder theoretischen maximalen Herzfrequenz stellt per se keinen Abbruchgrund dar.

\section{Durchführung der Ergometrie}

\subsection{Wahl des Belastungsprotokolls}

Grundsätzlich sollte sich das Belastungsprotokoll an der Leistungsfähigkeit des Patienten orientieren. Aus diesem Grund empfehlen internationale Gesellschaften [4, 5, 27-29] individuelle, der Leistungsfähigkeit angepasste Belastungsvorgaben.

Allgemeines Ziel ist es, in ca. 8-12 Minuten eine Ausbelastung zu erreichen. Belastungen von kürzerer Dauer führen nicht $\mathrm{zu}$ einer vollen kardiorespiratorischen Ausbelastung. Obwohl aus Untersuchungen hervorgeht, dass eine Belastungsdauer bis zu 17 Minuten zu keiner signifikanten Einschränkung der Maximalwerte führt [29], bringen Belastungszeiten über 12 Minuten keinen zusätzlichen Informationsgewinn. Darüber hinaus können länger dauernde Belastungen durch muskuläre Erschöpfung ebenfalls zu einem vorzeitigen Belastungsabbruch ohne kardiorespiratorische oder symptomlimierte Ausbelastung führen.

\begin{tabular}{ll}
\hline Tabelle 11: Kontraindikationen & \\
\hline Absolute Kontraindikationen & Relative Kontraindikationen \\
\hline Akutes Koronarsyndrom & Hauptstammstenose \\
Symptomatische Herzrhythmus- & Klappenerkrankungen mäßigen \\
störung und/oder eingeschränkte & Schweregrades \\
Hämodynamik & Bekannte Elektrolytstörungen \\
Symptomatische hochgradige & Arterielle Hypertonie \\
Aortenstenose & (RR > 180/100 mmHg) \\
Dekompensierte Herzinsuffizienz & Tachyarrhythmie oder Brady- \\
Akute Lungenembolie & arrhythmie \\
Akute Karditis (Endo-, Myo-, Peri- & Hypertrophe obstruktive \\
karditis) & Kardiomyopathie \\
Akute Aortendissektion & Höhergradige AV-Blockierungen \\
Fieberhafte Infekte & Anämie \\
Akute Phlebothrombose der & Physische und/oder psychische \\
unteren Extremität & Beeinträchtigungen \\
\hline
\end{tabular}

\begin{tabular}{|c|c|}
\hline Absolute Abbruchkriterien & Relative Abbruchkriterien \\
\hline $\begin{array}{l}\text { Abfall des systolischen Blutdrucks } \\
\text { um mindestens } 10 \text { mmHg gegen- } \\
\text { über dem Ausgangsblutdruck trotz } \\
\text { eines Anstiegs der Belastung mit } \\
\text { anderen Zeichen der Ischämie }\end{array}$ & \multirow[t]{2}{*}{$\begin{array}{l}\text { Abfall des systolischen Blut- } \\
\text { drucks um mind. } 10 \mathrm{mmHg} \\
\text { gegenüber dem Ausgangs- } \\
\text { blutdruck trotz eines Anstiegs } \\
\text { der Belastung ohne andere } \\
\text { Zeichen der Ischämie }\end{array}$} \\
\hline Eindeutige Angina pectoris & \\
\hline $\begin{array}{l}\text { Zunehmende zerebrale Sympto- } \\
\text { matik (z.B. Ataxie, Verwirrtheit, } \\
\text { Präsynkope) }\end{array}$ & \multirow{2}{*}{$\begin{array}{l}\text { ST- oder QRS-Veränderungen } \\
\text { wie horizontale oder deszen- } \\
\text { dierende ST-Senkung (>0,2 mV) } \\
\text { oder ausgeprägter Lagetyp- } \\
\text { wechsel }\end{array}$} \\
\hline Zeichen verminderter peripherer & \\
\hline Perfusion (Zyanose oder Blässe) & \multirow{3}{*}{$\begin{array}{l}\text { Arrhythmien anderer Art als } \\
\text { anhaltende ventrikuläre Tachy- } \\
\text { kardien, einschließlich multi- } \\
\text { fokaler ventrikulärer Extra- } \\
\text { systolen, Triplets, supraventri- } \\
\text { kuläre Tachykardien, Blockie- } \\
\text { rungen oder Vorhofflimmern }\end{array}$} \\
\hline $\begin{array}{l}\text { Technische Gründe, die es nicht } \\
\text { möglich machen, das EKG oder } \\
\text { den systolischen Blutdruck aus- } \\
\text { reichend auszuwerten }\end{array}$ & \\
\hline \multirow{3}{*}{$\begin{array}{l}\text { Der Wunsch des Untersuchten, } \\
\text { die Belastung zu beenden (hier } \\
\text { sollte der Untersuchende unbe- } \\
\text { dingt verbal intervenieren, wenn } \\
\text { keine anderen Indikationen zum } \\
\text { Belastungsabbruch vorliegen) }\end{array}$} & \\
\hline & $\begin{array}{l}\text { Erschöpfung, Luftnot, Giemen, } \\
\text { Beinkrämpfe oder Claudicatio }\end{array}$ \\
\hline & \multirow{3}{*}{$\begin{array}{l}\text { Entwicklung eines Schenkel- } \\
\text { blockbildes oder intraventriku- } \\
\text { läre Leitungsverzögerung, die } \\
\text { nicht von einer ventrikulären } \\
\text { Tachykardie zu unterscheiden } \\
\text { sind }\end{array}$} \\
\hline $\begin{array}{l}\text { Anhaltende ventrikuläre Tachy- } \\
\text { kardie }\end{array}$ & \\
\hline \multirow{4}{*}{$\begin{array}{l}\text { ST-Elevation um mindestens } \\
0,1 \mathrm{mV} \text { in Ableitungen ohne } \\
\text { pathologische Q-Wellen } \\
\text { (nicht aVR oder V1) }\end{array}$} & \\
\hline & Zunehmende Angina pectoris \\
\hline & $\begin{array}{l}\text { Abfall der Tretkurbel-Umdre- } \\
\text { hungszahl }<40 \mathrm{U} / \mathrm{min}\end{array}$ \\
\hline & $\begin{array}{l}\text { Arterielle Hypertonie } \\
\text { ( } 250 \mathrm{mmHg} \text { systolisch und/ } \\
\text { oder } 115 \mathrm{mmHg} \text { diastolisch) }\end{array}$ \\
\hline
\end{tabular}

Hingegen können Ergometrien mit einheitlicher Belastungssteigerung bei leistungsschwachen Patienten zu vorzeitigem Belastungssabbruch und bei leistungsstarken Patienten zu nicht erwünschten und unnötig langen Belastungszeiten führen.

Da zu hohe abrupte Steigerungen der Belastung vom Patienten subjektiv schlecht toleriert werden, bieten sich Steigerungen mit einminütigem Abstand an. Zusätzlich bieten Ergometrien mit einem geringeren Zeit- und Belastungsinkrement 


\begin{tabular}{lcc}
\hline \multicolumn{2}{l}{ Tabelle 13: Protokolle } \\
\hline $\begin{array}{l}\text { Erwartete } \\
\text { Maximalbelastung }\end{array}$ & $\begin{array}{c}\text { Belastungs- } \\
\text { protokoll }\end{array}$ & $\begin{array}{c}\text { Start- und } \\
\text { Erholungsbelastung }\end{array}$ \\
\hline mind. $240 \mathrm{~W}$ & $20 \mathrm{~W} / \mathrm{min}$ & $40 \mathrm{~W}$ \\
mind. $180 \mathrm{~W}$ & $15 \mathrm{~W} / \mathrm{min}$ & $30 \mathrm{~W}$ \\
mind. $150 \mathrm{~W}$ & $12 \mathrm{~W} / \mathrm{min}$ & $24 \mathrm{~W}$ \\
mind. $120 \mathrm{~W}$ & $10 \mathrm{~W} / \mathrm{min}$ & $20 \mathrm{~W}$ \\
mind. $90 \mathrm{~W}$ & $7 \mathrm{~W} / \mathrm{min}$ & $14 \mathrm{~W}^{*}(20 \mathrm{~W})$ \\
unter $70 \mathrm{~W}$ & $5 \mathrm{~W} / \mathrm{min}$ & $10 \mathrm{~W}^{*}(20 \mathrm{~W})$ \\
\hline * unter der technischen Vorraussetzung, dass das Ergometer für \\
$\begin{array}{l}\text { Belastungen unter } 20 \mathrm{~W} \text { geeicht ist, ansonsten ist eine Start- } \\
\text { belastung von 20 W möglich. }\end{array}$ \\
\hline
\end{tabular}

den Vorteil einer besseren leistungsdiagnostischen Auswertbarkeit.

Aus praktischen Gründen empfiehlt es sich, das Belastungsinkrement entsprechend der zu erwartenden Maximalleistung (Tab. 13) anzugleichen [30].

Als Start- sowie Erholungsbelastung wird das Doppelte des Belastungsinkrementes empfohlen (Abb. 4).

Analog zum Fahrradtest werden beim Test auf dem Laufband ebenfalls individuell abgestimmte Protokolle empfohlen. Dabei bieten sich Steigerungen in 1-minütigem Abstand entweder der Geschwindigkeit (z. B. $5 \mathrm{~km} / \mathrm{h}+0,3-0,5 \mathrm{~km} / \mathrm{h} / \mathrm{min}$, Steigung $1^{\circ}$ konstant) oder der Steigung (z. B. $3^{\circ}+1 \% \mathrm{~min}$, $4 \mathrm{~km} / \mathrm{h}$ konstant) an. Alternativ können standardisierte Protokolle wie das Bruce- oder das modifizierte Bruce-Protokoll verwendet werden.

\subsection{Durchführung}

\subsubsection{Voraussetzungen}

Siehe bei 2 .

\subsubsection{Vorbereitung}

Ruhiges Sitzen am Ergometer für 3 Minuten, danach RRMessung. Im Anschluss Beginn der Belastungsphase entsprechend dem Belastungsprotokoll.

\subsubsection{Belastungs- und Nachbelastungsphase}

Die Belastungssteigerung erfolgt entsprechend dem Belastungsprotokoll, die Umdrehungszahl am Fahrradergometer sollte sich in einem Bereich von 60-90 Umdrehungen/ min bewegen, in Einzelfällen (z. B. Sportuntersuchungen) können auch höhere Umdrehungszahlen zugelassen werden. Die Trittfrequenz sollte aber über die gesamte Untersuchungsdauer konstant $( \pm 5$ Umdrehungen $/ \mathrm{min})$ gehalten werden.

Kontinuierliche klinische Überwachung (wie in den Punkten 2 (Voraussetzungen) und 11 (Dokumentation) angeführt); die Herzfrequenz- und EKG-Aufzeichnung erfolgt kontinuierlich, eine EKG-Dokumentation und RR-Messung mindestens alle 2 min. Weiters erfolgt eine kontinuierliche Deskription allfälliger Rhythmusstörungen.

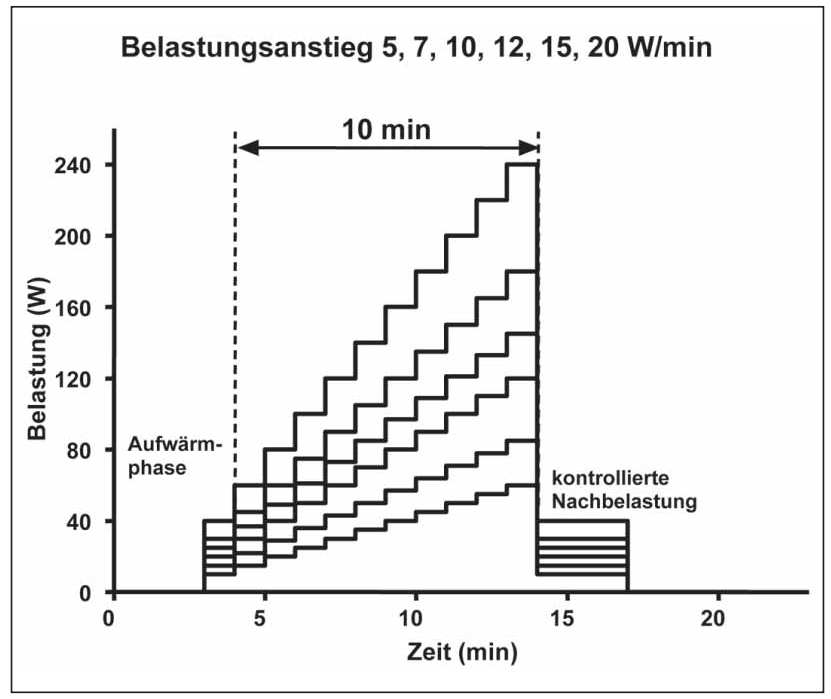

Abbildung 4: Belastungsprotokoll. Nachdruck mit freundlicher Genehmigung von P. Hofmann [30].

\begin{tabular}{llll}
\hline \multicolumn{2}{l}{ Tabelle 14: PER (Percieved Exertion Rating) Skala } \\
\hline 7 & Sehr sehr leicht & 15 & Schwer \\
9 & Sehr leicht & 17 & Sehr schwer \\
11 & Mässig leicht & 19 & Sehr sehr schwer \\
13 & Etwas schwer & & \\
\hline
\end{tabular}

\begin{tabular}{llll}
\hline Tabelle 15: A.P.-Skala & & \\
\hline 0 & Kein Schmerz & III & Leichter Schmerz \\
I & Sehr geringer Schmerz & IV & Starker Schmerz \\
II & Geringer Schmerz & V & Sehr starker Schmerz \\
\hline
\end{tabular}

\begin{tabular}{|c|c|c|c|}
\hline \multicolumn{4}{|c|}{ Tabelle 16: Symptom-Skala } \\
\hline 1 & Allgemeine Erschöpfung & 8 & Blässe \\
\hline 2 & Schwäche der Beine & 9 & Zyanose \\
\hline 3 & Beinschmerzen & 10 & Schlecht motiviert \\
\hline 4 & Dyspnoe & 11 & Braucht Nitro \\
\hline 5 & Müdigkeit & 12 & Braucht $\mathrm{O}_{2}$ \\
\hline 6 & Schwindelgefühl & 13 & Kreislaufkollaps \\
\hline 7 & Kalter Schweiß & 14 & Anderes \\
\hline
\end{tabular}

Optional: Kontinuierliche Messung der Atemgase, Blutlaktatbestimmung am Ende jeder Belastungsstufe

\section{Bewertung der Belastungsuntersuchung}

\subsection{Klinische Symptomatik (Tab. 14-16)}

Typische Angina pectoris wird auch ohne entsprechende EKG-Veränderungen in $30 \%$ der Fälle als alleiniges Zeichen einer myokardialen Ischämie angegeben [6].

\subsection{Körperliche Leistungsfähigkeit}

Eine wesentliche Ergebnisgröße der Ergometrie ist die erbrachte Leistung auf dem Ergometer. In der internationalen Literatur richtet sich die individuelle Beurteilung der Leistungsfähigkeit nach dem Vielfachen des Energieumsatzes in Ruhe (= metabolisches Äquivalent MET). Da die direkte Be- 


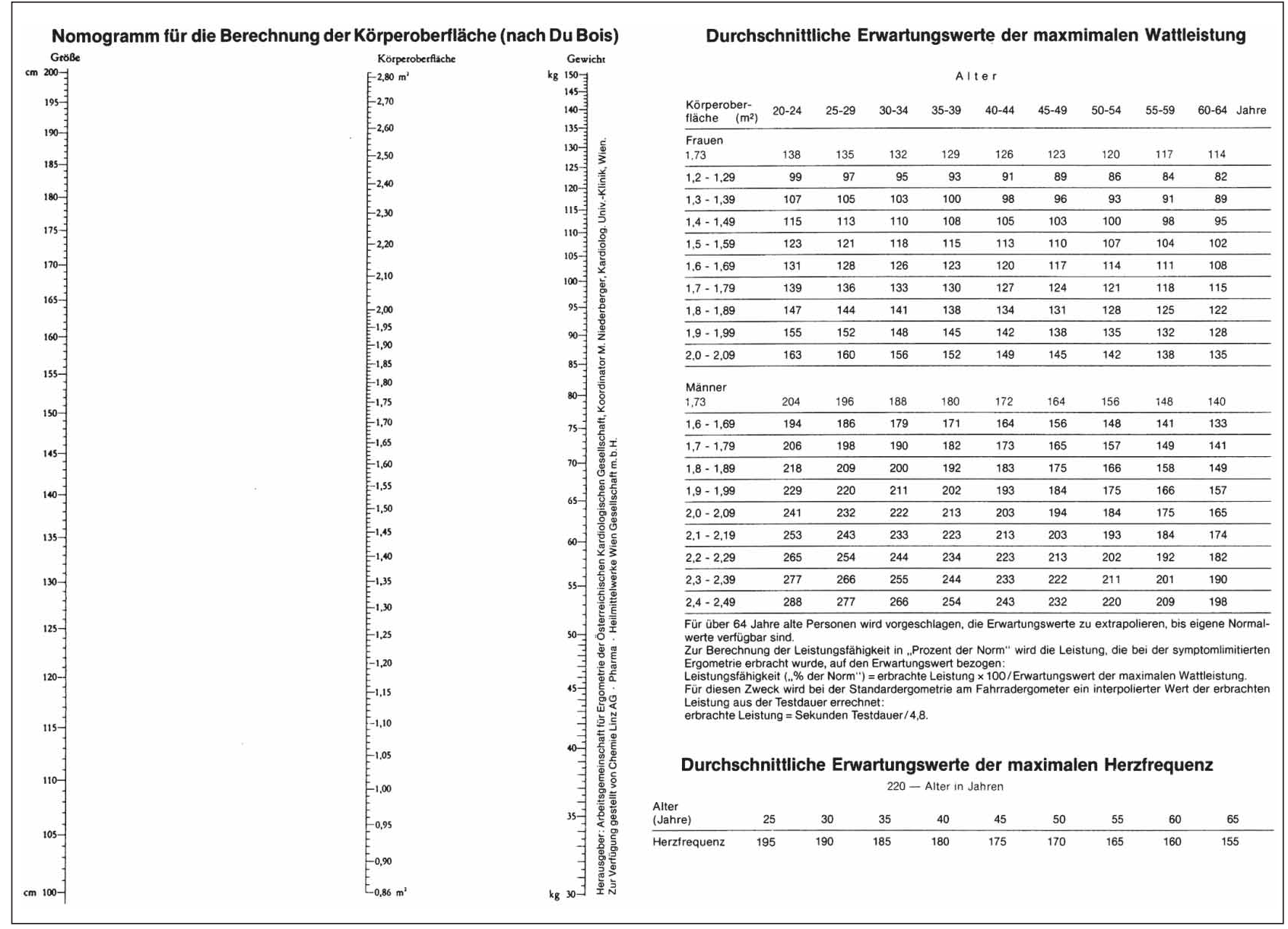

Abbildung 5: Sollwerte für Fahrradergometrie

stimmung mittels Spiroergometrie ein aufwändiges Verfahren darstellt, wird üblicherweise eine indirekte Berechnung aus den Leistungsdaten der Laufband- bzw. Fahrradergometrie herangezogen.

Die Beurteilung der Leistungsfähigkeit setzt eine maximale Ausbelastung voraus, dies ist jedoch nicht gleichzusetzen mit dem Erreichen einer theoretischen maximalen Herzfrequenz. Einflussgrößen auf die Leistungsfähigkeit sind Alter, Größe, Gewicht und Umgebungsbedingungen.

Für die Berechnung der tatsächlich erreichten Leistung wird ein interpolierter Wert errechnet:

Erbrachte Leistung $(\mathrm{W})=$ Leistung der letzten vollendeten Stufe $(\mathrm{W})+($ Steigerungshöhe $(\mathrm{W}) \times$ Dauer der letzten Stufe (s) / vorgegebene Stufendauer (s).

Obwohl die Normwerte aus der Literatur teilweise differieren, sollte als grober Anhaltspunkt ein Vergleich mit einem Sollwert (Abb. 5) erfolgen.

Dieser kann aus Abbildung 5 ermittelt oder nach folgender Formel berechnet werden:

Körperoberfläche KO $\left(\mathrm{m}^{2}\right)=0,007148 \times \mathrm{KG}(\mathrm{kg})^{0,425} \times$ $\mathrm{L}(\mathrm{cm})^{0,725}$
Männlich:

Leistung $(\mathrm{W})=6,773+136,141 \times \mathrm{KO}-0,916 \times \mathrm{KO} \times \mathrm{A}$ (Jahre)

Weiblich:

Leistung $(\mathrm{W})=3,933+86,641 \times \mathrm{KO}-0,346 \times \mathrm{KO} \times \mathrm{A}$

(Jahre)

Die Leistungsfähigkeit stellt per se einen eigenständigen prognostischen Faktor hinsichtlich der Gesamtmortalität dar.

Zur Beurteilung einer etwaigen Arbeitsfähigkeit (Begutachtung) sind neben der körperlichen Leistungsfähigkeit unbedingt auch weitere klinische Parameter sowie individuelle Arbeitsbedingungen heranzuziehen.

\subsection{Herzfrequenz}

Die Herzfrequenz zeigt bei Herzgesunden typischerweise einen nicht ganz linearen, s-förmigen Verlauf [15]. Die maximale Herzfrequenz nimmt linear mit dem Alter ab, der individuelle Maximalwert ist aber einer großen Streuung unterworfen. So existieren mehrere Formeln zur Berechnung der maximalen Herzfrequenz, alle jedoch mit einer entsprechend hohen Standardabweichung. Daher wird empfohlen, sich eine einfache Formel zu merken:

$$
\mathrm{HF}_{\max }=220-\text { Alter }( \pm 15 \%)
$$


Ein verzögerter Herzfrequenzanstieg und/oder das Nichterreichen des unteren Erwartungswertes kann medikamentös induziert (z. B. Betablocker), aber auch Zeichen einer Sinusknotenfunktionsstörung sein. Eine sog. „chronotrope Inkompetenz", bei der weniger als $85 \%$ der erwarteten maximalen Herzfrequenz erreicht wird, geht bei Patienten mit bekannter Herzerkrankung ohne bradykardisierende Medikation mit einer erhöhten Mortalität einher [31]. Der Herzfrequenzverlauf ist bei Patienten mit KHK meist derart verändert, dass es nach einem anfänglichen verzögerten Herzfrequenzanstieg ab einer Belastung von ca. $75 \%$ der Maximalbelastung zu einem überschießendem Anstieg der Herzfrequenz als Zeichen einer myokardialen Einschränkung der Pumpfunktion im anaeroben Bereich der Energieproduktion kommt (Abb. 2) [8, 10].

\subsection{Blutdruckverhalten}

Der systolische Blutdruck steigt unter zunehmender Belastung kontinuierlich an. Unter physiologischen Bedingungen sollte der systolische Blutdruck um $5-10 \mathrm{mmHg}$ pro MET (ca. 20-30 W) ansteigen [16].

Der Blutdruck sollte bei untrainierten gesunden Personen unter maximaler Belastung systolisch nicht über $250 \mathrm{mmHg}$ oder nicht mehr als $140 \mathrm{mmHg}$ über den Ausgangswert erreichen [16]. Allerdings sind bei ausdauertrainierten, leistungsfähigeren Personen in Abhängigkeit der Maximalleistung auch deutlich höhere systolische Blutdruckwerte durchaus physiologisch. Eine Belastungshypertonie oder unzureichend medikamentös behandelte arterielle Hypertonie liegt daher dann vor, wenn der Blutdruck, gemessen bei der Fahrradergometrie, auf einer gegebenen Wattstufe, den nach der Formel

$$
\text { RRsyst }=120+0,4 \times(\text { Watt }+ \text { Lebensalter in Jahren })
$$

errechneten Wert übersteigt [32]. Liegt eine Belastungshypertonie vor, so ist diese ein Vorzeichen für eine zukünftige Hypertonie [33-35]. Darüber hinaus ist die Belastbarkeit durch eine pathologische Blutdruckregulation eingeschränkt [36] und eine hochgradige hypertensive Blutdruckregulation kann unter Belastung auch zu ischämischen EKG-Veränderungen ohne Arteriosklerose führen [37]. Mittel- und langfristig geht eine Belastungshypertonie allerdings mit einem gesteigerten Risiko der Entwicklung eines Myokardinfarktes einher [38].

Patienten mit einer eingeschränkten Pumpfunktion unter Belastung reagieren mit einer unzureichenden Steigerung des systolischen Blutdrucks. Unzureichende systolische Blutdruckanstiege (maximaler systolischer Blutdruck $<120 \mathrm{mmHg}$ ) und ein signifikanter Blutdruckabfall unter den Ausgangswert sind als Zeichen einer schweren myokardialen Pumpfunktionsstörung und/oder einer koronaren Mehrgefäßerkrankung zu werten. Ein sehr schneller Blutdruckabfall nach Belastungsbeginn kann auf eine Hauptstammstenose hinweisen. Andere Ursachen von Blutdruckabfällen sind supraventrikuläre oder ventrikuläre Arrhythmien, vasovagale Reaktionen, linksventrikuläre Ausflussbahnobstruktionen, Wirkung von Antihypertensiva und Hypovolämie [38]. Auf jeden Fall sollte ein Abfall des systolischen Blutdruckes auf Werte unter den Ausgangswert oder ein signifikanter Abfall des

\begin{tabular}{|c|c|c|}
\hline & Physiologisch & Pathologisch \\
\hline P-Welle & $\begin{array}{l}\text { P-Überhöhung in } \\
\text { II, III, avF }\end{array}$ & Abnahme der P-Welle \\
\hline PQ-Zeit & Verkürzung & Verlängerung \\
\hline Q-Zacke & & $\begin{array}{l}\text { Bereits in Ruhe patho- } \\
\text { logisch }\end{array}$ \\
\hline R-Zacke & $\begin{array}{l}\text { Anfängliche Zunahme, } \\
\text { danach Abnahme links- } \\
\text { präkordial }\end{array}$ & $\begin{array}{l}\text { Permanente Zunahme } \\
\text { häufig bei ischämischen } \\
\text { Reaktionen }\end{array}$ \\
\hline S-Zacke & Zunahme präkordial & \\
\hline \multirow[t]{2}{*}{ QRS-Komplex } & $\begin{array}{l}\text { Verschiebung des R/S- } \\
\text { Überganges nach links }\end{array}$ & Verbreiterung \\
\hline & Änderung des Lagetyps & \\
\hline ST-Strecke & $\begin{array}{l}\text { Absenkung des J-Punktes } \\
\text { mit rasch aszendierender } \\
\text { (>1mV/sek) ST-Strecke }\end{array}$ & $\begin{array}{l}\text { ST-Hebungen; } \\
\text { ST-Senkungen (deszen- } \\
\text { dierend, horizontal, träg } \\
\text { aszendierend) }\end{array}$ \\
\hline T-Welle & Abflachung & Zunahme $>1 \mathrm{~mm}$ \\
\hline QT-Dauer & Verkürzung & Verlängerung \\
\hline
\end{tabular}

systolischen Blutdrucks um mehr als $10 \mathrm{mmHg}$ nach anfänglicher Blutdrucksteigerung abhängig von der klinischen Symptomatik u/o Ischämiezeichen zum Abbruch der Ergometrie führen (Tab. 12).

In der unmittelbaren Nachbelastungsphase kommt es $\mathrm{zu}$ einem unverzüglichen Abfall des systolischen Blutdrucks. Der systolische Blutdruck sollte 3 Minuten nach Belastungsabbruch weniger als $90 \%$ des Wertes unter maximaler Belastung betragen. Sollte es nicht möglich sein, den Blutdruck bei maximaler Belastung zu messen, so sollte der Blutdruck in der dritten Nachbelastungsminute deutlich niedriger als in der ersten Nachbelastungsminute sein [16]. In jedem Fall geht eine verzögerte Blutdrucknormalisierung in der Nachbelastungsphase mit einem zunehmenden Risiko für koronare Herzkrankheit und einer ungünstigen Prognose einher [39].

\subsection{EKG}

Eine Reihe physiologischer wie auch pathologischer EKGVeränderungen sind beschrieben [5] (Tab. 17).

Eine besondere Bedeutung in der Interpretation des EKGs zur Ischämiediagnostik unter Belastung kommt der Analyse der ST-Strecke zu. Als Bezugspunkt wird der J-Punkt, das ist derjenige Punkt, wo die S-Zacke in die ST-Strecke übergeht, herangezogen (Abb. 6).

Veränderungen der ST-Strecke lassen sich zunächst in STSenkungen und ST-Hebungen unterteilen. ST-Senkungen werden wiederum in aszendierend, horizontal und deszendierend unterteilt (Abb. 7).

Aszendierende ST-Strecken-Senkungen werden v. a. bei rasch aszendierendem Verlauf (>1 mV/sek) als normal angesehen. Sollte jedoch der Verlauf nur träg aszendierend sein oder eine ausgeprägte ST-Senkung ( $>0,15 \mathrm{~ms}$ 60-80 ms nach dem J-Punkt) vorhanden sein, gilt auch dies als Hinweis für eine Belastungskoronarinsuffizienz. 
Horizontale oder deszendierende ST-Strecken-Senkungen unter Belastung (Abb. 6) lassen jedoch auf ein pathologisches Korrelat schließen. Die nachfolgende ST-Strecke muss für 60-80 msec horizontal oder deszendierend gesenkt sein. Sollte diese Senkung 60-80 msec nach dem J-Punkt > 0,1 mV betragen, so besteht der hochgradige Verdacht auf eine Belastungskoronarinsuffizienz.

Die Zunahme einer schon in Ruhe vorbestehenden STStreckensenkung von $>0,1 \mathrm{mV}$ gilt ebenfalls als pathologisch.

Allerdings muss auch die Normalisierung einer in Ruhe vorhandenen ST-Senkung unter Belastung und gleichzeitigen Beschwerden als Pseudonormalisierung angesehen und der Verdacht auf Ischämie erhoben werden. Je ausgeprägter ST-Strecken-Senkungen unter Belastung sind (z. B. ST-Senkung $>0,2 \mathrm{mV}$, deszendierender ST-Streckenverlauf, ST-Strecken-Senkungen in $>5$ Ableitungen und Persistenz der STSenkungen noch 5 Minuten nach Belastungsende), umso höher ist die Wahrscheinlichkeit einer KHK und umso ausgeprägter sind die Koronarveränderungen.

Die ST-Strecken-Senkung erlaubt keine sichere Lokalisation des Ortes der Ischämie, somit ist eine anatomische Zuordnung zum Koronargefäß nur eingeschränkt möglich. Bei Patienten mit Linksschenkelblock ist die Bewertung der ST-Strecke unter Belastung nicht möglich, wohingegen eine Bewertung der linksgerichteten Ableitungen beim Rechtsschenkelblock möglich ist.

Hebungen der ST-Strecke unter Belastung $>0,1 \mathrm{mV}$ im Bereich des J-Punktes bzw. 60-80 ms nach dem J-Punkt sind bei Auftreten in jeweils 3 aufeinander folgenden EKG-Komplexen als Zeichen einer transmuralen Ischämie zu werten und müssen zu einem Belastungsabbruch führen. Eine Ausnahme stellen auftretende ST-Hebungen in Ableitungen mit vorbestehender pathologischer Q-Zacke nach abgelaufenem Myokardinfarkt dar. Hier findet sich oft eine ST-Hebung als Ausdruck einer dyskinetischen linksventrikulären Wandbewegungsstörung.

Eine T-Wellenabflachung unter Belastung ist physiologisch. T-Wellen-Hebungen von $>1 \mathrm{~mm}$ in Ableitungen ohne Q-Zacke sind als pathologisch zu werten und gehen mit einer schweren KHK und schlechten Prognose einher. Ein negatives T vor Belastung mit Aufrichtung („Pseudonormalisierung“) während der Belastung mit entsprechender Klinik sollte als pathologisch (Ischämie) gewertet werden. Bei jüngeren Menschen und bei Ausdauersportlern kann jedoch ein Aufrichten der negativen T-Welle Ausdruck eines Sportherzens (exzentrische linksventrikuläre Hypertrophie) sein.

\subsubsection{Herzrhythmusstörungen}

Ventrikuläre Rhythmusstörungen, die nur unter Belastung auftreten, gehen nicht mit einem erhöhten Mortalitätsrisiko einher. Nichtsdestotrotz ist eine weitere Abklärung individuell anzustreben. Eine erhöhte Mortalität findet sich hingegen bei Auftreten von ventrikulären Rhythmusstörungen nach Belastungsende bzw. eine weitere Mortalitätszunahme bei Auftreten während und nach der Belastung [40, 41].

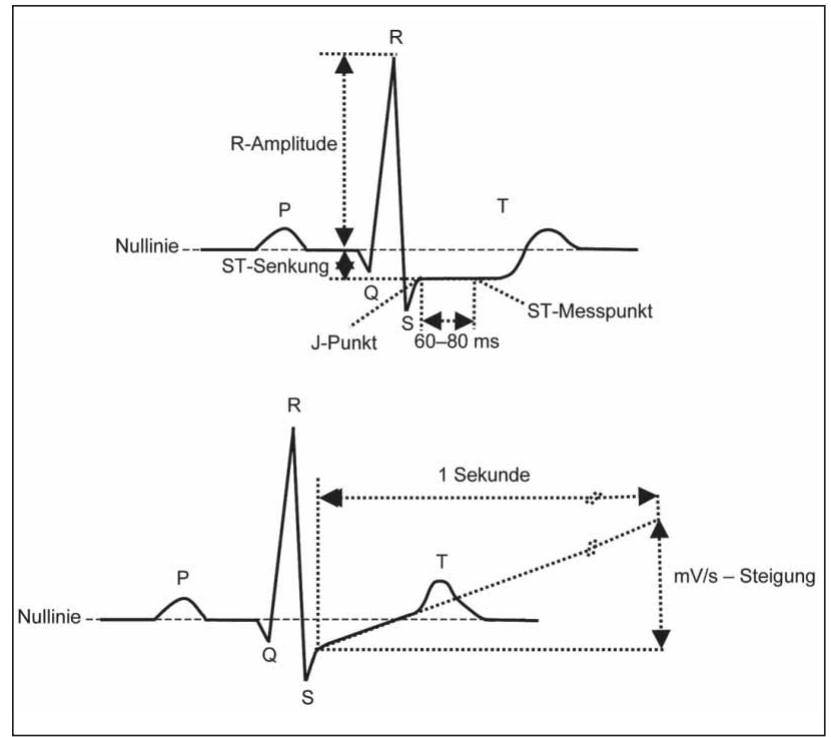

Abbildung 6: ST-Strecke
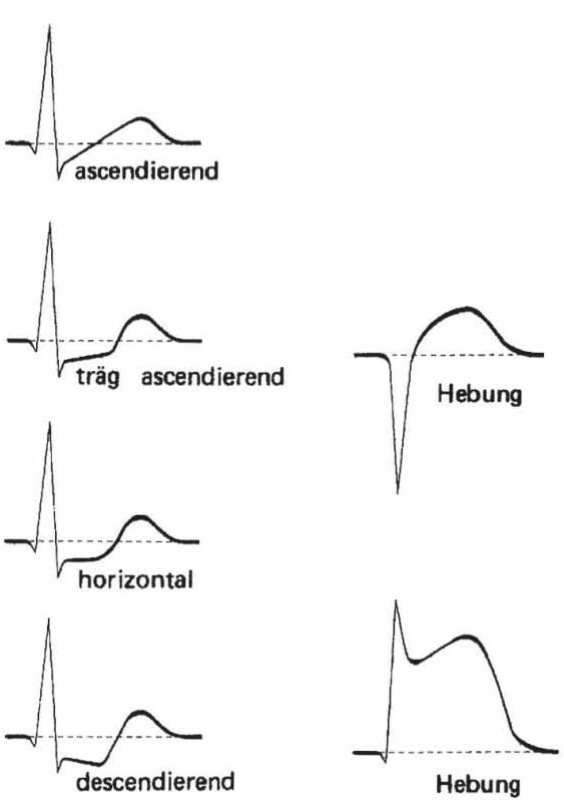

Abbildung 7: ST-Streckenveränderungen

Das Auftreten von Erregungsleitungsstörungen unter Belastung ist als pathologisch zu werten und bedarf einer weiteren Abklärung. Ebenfalls kann das Auftreten von Vorhofflimmern unter Belastung durch eine Ischämie ausgelöst werden, wenngleich die häufigste Ursache das Bestehen einer Hypertonie darstellt.

Das Auftreten eines Linksschenkelblockes unter Belastung ist als pathologisch zu werten und kann neben einer ischämischen Genese auch degenerative Veränderungen des Reizleitungssystems zu Grunde haben.

7.5.2. Wertigkeit des Belastungs-EKGs für die Ischämiediagnostik

Die Sensitivität ist ein $\mathrm{Ma}$ dafür, wie viele richtige positive Befunde ein Test liefert, verglichen mit der Anzahl aller 


\begin{tabular}{|c|c|c|c|c|}
\hline & $n$ & $\begin{array}{c}\text { Sensitivität } \\
(\%)\end{array}$ & $\begin{array}{c}\text { Spezifität } \\
(\%)\end{array}$ & $\begin{array}{l}\text { Vorhersage- } \\
\text { wert (\%) }\end{array}$ \\
\hline & \multicolumn{4}{|c|}{ Geschlecht } \\
\hline Männer & 1465 & 80 & 74 & 88 \\
\hline \multirow[t]{2}{*}{ Frauen } & 580 & 76 & 64 & 46 \\
\hline & \multicolumn{4}{|c|}{ Ruhe-EKG } \\
\hline normal & 1528 & 76 & 77 & 81 \\
\hline abnormal & 517 & 88 & 43 & 72 \\
\hline
\end{tabular}

Erkrankten. Die Spezifität gibt an, wie viele Patienten vom Test als richtig gesund erkannt werden, gemessen an der Anzahl aller Gesunden. Der positive Vorhersagewert gibt an, wie viele richtig positive Befunde ein Test liefert verglichen mit der Anzahl der richtig und falsch positiven Befunde (Tab. 18).

\section{Leistungsdiagnostik und Trainings- therapie}

Die Wirksamkeit einer Trainingstherapie ist abhängig von der Trainingsintensität. Um eine Überlastung sowohl des kardiorespiratorischen als auch metabolischen Systems zu vermeiden [10, 42, 43], steht eine aerobes Ausdauertraining im Vordergrund. Aus diesem Grund ist eine Belastungsobergrenze unter der anaeroben Schwelle einzuhalten $[8,10,42-$ 44]. Hingegen verhindert eine zu geringe Trainingsintensität mit Belastungen unterhalb der aeroben Schwelle ein therapeutisch optimal wirksames Training [44, 45] (Abb. 2).

Verschiedene Methoden zur Ermittlung der aeroben und anaeroben Schwelle unter Verwendung der Atemgasanalyse, der Blutlaktatkonzentration oder der Herzfrequenz sind in der Literatur beschrieben [11, 12, 45-48].

\subsection{Laktatleistungsdiagnostik}

Zur Bestimmung der aeroben und anaeroben Schwelle werden entsprechend der Dreiphasigkeit der Energiebereitstellung die beiden Umstellpunkte aus der Lakat-Leistungskurve (,lactate turn points“) empfohlen (Abb. 2) [8, 11, 46, 48-51].

\subsection{Atemgasanalyse}

Analog zum Verhalten der Laktat-Leistungskurve ergeben sich Umstellpunkte in den ventilatorischen Parametern, eine erste ventilatorische Schwelle (= ,ventilatory threshold“, VT1) entsprechend der aeroben Schwelle und eine zweite ventilatorische Schwelle (VT2) entsprechend der anaeroben Schwelle [12, 45, 46, 48, 52].

\subsection{Herzfrequenzleistungsdiagnostik}

Kann eine Krümmung der Herzfrequenzleistungskurve, unabhängig von der Richtung, nachgewiesen werden, kann ein Herzfrequenzdeflexionspunkt (= ,heart rate turn point“, HRTP), der dem Übergang von Phase II zu Phase III der Ener- giebereitstellung entspricht, berechnet und zur Leistungsdiagnostik herangezogen werden $[14,15,52-54]$ (s. 7.3.1.). Nimmt jedoch die Beziehung zwischen HF und Leistung einen annähernd linearen Verlauf, so liegt der Übergang zwischen Phase II und Phase III der Energiebereitstellung bei ca. $70 \%$ der Herzfrequenzreserve. Nur in diesen Fällen sollte die Herzfrequenzreserve [55] zur Trainingssteuerung herangezogen werden $[46,47,54]$ (s. 8.4.3.).

\subsection{Trainingsvorschreilbung}

In der Praxis wird oft die Anforderung an den Arzt in der Erstellung einer groben Trainingsherzfrequenz gestellt. Für ein präventives und rehabilitatives Ausdauertraining wird die Trainingsintensität durch 3 relativ einfache Methoden festgelegt.

8.4.1. Herzfrequenz bei $80-90 \%$ der Herzfrequenz an der anaeroben Schwelle

8.4.2. Herzfrequenz bei $\mathbf{5 0 - 7 0 \% ~ d e r ~ m a x i m a l e n ~ W a t t l e i s - ~}$ tung

Einfachstes Verfahren, es ist jedoch eine Ausbelastung erforderlich.

8.4.3. Herzfrequenz bei $50-70 \%$ der Herzfrequenzreserve Die Trainingsherzfrequenz wird folgendermaßen berechnet:

$$
\mathrm{HF}_{\text {Training }}=\mathrm{HF}_{\text {Ruhe }}+\left(\left(\mathrm{HF}_{\max }-\mathrm{HF}_{\text {Ruhe }}\right) \times 0,5-0,7\right)
$$

Voraussetzung für diese Berechnung ist ein linearer Verlauf der Herzfrequenz bis zum Maximum sowie die vollständige Ausbelastung.

\section{Komplikationen (Tab. 19)}

Klassifizierung von Patienten mit deutlich erhöhtem Risiko für kardiale Komplikationen während der Belastungsuntersuchung [96]:

Nach Diagnose:

1. Koronare Herzerkrankung

2. Herzklappenerkrankung mit Ausnahme einer hochgradigen Stenose oder Insuffizienz

3. Kongenitale Herzerkrankung [93]

4. Kardiomyopathie mit EF $<30 \%$ bei stabiler klinischer Situation mit Ausnahme einer HCM oder Myokarditis

5. Rezidivierende ventrikuläre Tachykardien unter Therapie

Nach Klinik:

1. NYHA 3 oder 4

2. Belastungsuntersuchung: Leistungsfähigkeit von < 6 METs; Angina pectoris oder ischämische ST-Veränderungen bei geringer Belastung, < 6 METs nicht anhaltende ventrikuläre Tachykardien unter Belastung

3. Füherer Herzstillstand ohne Assoziation mit einem akuten Myokardinfarkt oder einer invasiven kardialen Untersuchung

4. Ein allgemeines medizinisches Problem, welches lebensbedrohend sein könnte 


\begin{tabular}{|c|c|c|}
\hline Ereignis & Häufigkeit & Kommentar \\
\hline \multicolumn{3}{|l|}{ Kardial } \\
\hline Morbidität & $<0,05 \%$ & {$[56,70]$} \\
\hline Tödliche Komplikationen & $0,03-0,04 \%$ & häufiger bei symptomlimitierter als bei submaximaler Belastung [57-59] \\
\hline Nicht tödliche Komplikationen & $0,07-0,15 \%$ & häufiger bei symptomlimitierter als bei submaximaler Belastung [57-59] \\
\hline Akuter $\mathrm{MCl}$ & $0,035-0,1 \%$ & $\begin{array}{l}4-20 \% \text { der } \mathrm{MCl} \text { während oder in } 1 \text {. Stunde nach Belastung } \\
\mathrm{MCl} 7 \times \text { häufiger als plötzlicher Herztod [5, 60-62] }\end{array}$ \\
\hline Plötzlicher Herztod & $<0,005 \%$ & [64] \\
\hline Ventrikuläre Tachyarrhythmien & $0,05-2,3 \%$ & bei hohem Risiko für ventrikuläre Arrhythmien bis 2,3 \% [73, 74] \\
\hline Supraventrikuläre Tachyarrhythmien & $\begin{array}{l}3,4-15 \% \\
<1 \%\end{array}$ & $\begin{array}{l}\text { bei bekannten paroxysmalen supraventrikulären Arrhythmien [70, 75-77, 94, 95] } \\
\text { Vorhofflimmern/-flattern [5] }\end{array}$ \\
\hline Ventrikuläre Extrasystolie & $2-20 \%$ & $\begin{array}{l}\text { Bei bekannter KHK in 7-20 \%; 2-8 \% bei asymptomatischen Patienten } \\
{[4,18,19,34]}\end{array}$ \\
\hline Supraventrikuläre Extrasystolie & $4-24 \%$ & {$[70,75-77,94,95]$} \\
\hline Paroxysmales Vorhofflimmern & $0,8 \%$ & [94] \\
\hline Passagerer Linksschenkelblock & $0,4 \%$ & Unabhängiger Risikofaktor für Tod und kardiovaskuläres Ereignis [93] \\
\hline Bradyarrhythmien & & $\begin{array}{l}\text { Sick-Sinus-Syndrom, höhergradige AV-Blockierungen, chronotrope Inkompetenz, } \\
\text { PM Dysfunktion [78-84] }\end{array}$ \\
\hline Hypotonie & $3-9 \%$ & $\begin{array}{l}\text { bei koronarer Mehrgefäßerkrankung oder Hauptstammstenose, Kardiomyopathie } \\
\text { mit hochgradig reduzierter Linksventrikelfunktion, linksventrikuläre Ausflusstrakt- } \\
\text { obstruktion, Herzklappenerkrankung, Arrhythmien, vasovagale Reaktion, Anti- } \\
\text { hypertensiva, Volumenmangel [85-90] }\end{array}$ \\
\hline \multicolumn{3}{|l|}{ Hypertensive Blutdruckregulation } \\
\hline Ventrikelruptur & & sehr seltenes Ereignis nach $\mathrm{MCl}[65,66]$ \\
\hline Papillarmuskelabriss & & sehr seltenes Ereignis nach $\mathrm{MCl}$ [67] \\
\hline
\end{tabular}

\section{Nicht kardial}

Intrakranielle Blutung [68], thromboembolisches Ereignis [69], transiente globale Amnesie [91]

Gelenksbeschwerden, Muskelschmerzen, Lumbalgie

\section{Einflussgrößen}

\subsection{Geschlecht}

Sowohl die Sensitivität als auch die Spezifität der Belastungsuntersuchung ist vor allem bei submaximaler Belastung wegen einer geringeren Prävalenz der koronaren Herzkrankheiten besonders bei prämenopausalen Frauen und auch wegen der geringeren Vortestwahrscheinlichkeit im Vergleich zu Männern niedriger (Tab. 18) [96].

Während der Ergometrie geben Frauen weniger häufig typische Angina pectoris-Symptome an und beschreiben ihre Beschwerdesymptomatik sehr unterschiedlich, oft jedoch sehr heftig und verspüren öfter unspezifische kardiale Beschwerden [97].

Auch die geringere Sensitivität der belastungsinduzierten EKG-Veränderungen, insbesondere der ST-Strecken-Senkungen, die möglicherweise auch mit der höheren Prävalenz des Mitralklappenprolapses und der mikrovaskulären Funktionsstörung der Koronargefäße bei der Frau in Zusammenhang stehen, schränkt die Aussagekraft dieser Untersuchung bei der Frau ein [98].

\subsection{Medikamente}

Medikamente beeinflussen die Aussagekraft von Belastungsuntersuchungen, daher ist im Vorfeld genau zu prüfen, welche in Abhängigkeit zur Indikation der Belastungsuntersuchung eingenommen werden sollen.

\subsubsection{Betablocker}

Durch die reduzierte Herzfrequenzregulation kann bei KHKPatienten die Ischämieschwelle bei einer höheren Belastungsstufe erreicht und damit die Diagnostik beeinträchtigt werden. Ein Absetzen des Betablockers sollte jedoch in jedem Falle vor der Untersuchung genau überlegt werden, um nicht den Patienten unnötig den Problemen eines möglichen Reboundphänomens, wie AP-Symptomatik oder hypertone Blutdruckwerte, auszusetzen.

\subsubsection{Digitalis}

Bereits beim Koronargesunden kommt es unter Digitalismedikation in $25-40 \%$ bei Belastung zu ST-Strecken-Senkungen [99]. Daher ist unter Digitalistherapie eine Beurteilung des Belastungs-EKG's zur Diagnose der koronaren Herzkrankheit nur eingeschränkt möglich.

\subsubsection{Nitrate, Kalziumantagonisten}

Beide Substanzgruppen erhöhen aufgrund der antianginösen Wirkung die Ischämieschwelle und beeinflussen damit das Untersuchungsergebnis.

\subsubsection{Antiarrhythmika}

Belastungsinduzierte ventrikuläre Arrhythmien wurden unter einer Therapie mit Antiarrhythmika der Klasse Ic, vor allem unter Flecainid, beobachtet [100].

\subsection{Alter}

Abhängig vom Alter steigen Prävalenz und Schwere der Koronarstenosen, wodurch sich eine höhere Sensitivität 


\begin{tabular}{l} 
Tabelle 20: Mögliche Ursachen eines falsch positiven Be- \\
lastungs-EKG's \\
\hline Kardiale Ursachen \\
\hline Linkshypertrophie (Aortenvitium, hypertensive Krise) \\
Kardiomyopathie \\
Mitralklappenprolaps \\
WPW-Syndrom \\
Myokarditis \\
Ruhetachykardie \\
ST-Streckenveränderungen > 0,05 mV in Ruhe \\
Linksschenkelblock \\
Erkrankungen des Perikards \\
Nach Revaskularisation einer KHK bis zu 3-6 Monate \\
\hline Extrakardiale Ursachen \\
\hline Medikamente (Diuretika, Antiarrhythmika, Digitalis, Psychophar- \\
maka, Katecholamine, Hormone) \\
Hypokaliämie \\
Hypertonie \\
Schilddrüsenfunktionsstörungen (Hyper- und Hypothyreose) \\
Geschlecht (Frauen) \\
Anämie \\
Hyperventilation
\end{tabular}

\begin{tabular}{l} 
Tabelle 21: Mögliche Ursachen eines falsch negativen Be- \\
lastungs-EKG's \\
\hline Mangelnde Ausbelastung \\
Rechtsschenkelblock, Linksschenkelblock \\
Linksanteriorer Hemiblock \\
Rechtshypertrophie \\
Medikamente (Betablocker, Kalziumantagonisten, Nitrate, \\
Nicorandil, Molsidomin) \\
Koronare Eingefäßerkrankung \\
Schrittmacher (Ventrikelstimulation) \\
\hline
\end{tabular}

\begin{tabular}{ll}
\hline Tabelle 22: Dokumentation & \\
\hline Vor der Belastung & \\
\hline $\begin{array}{l}\text { Klinische Symptomatik } \\
\text { Diagnose und Fragestellung } \\
\text { Patientendaten }\end{array}$ & $\begin{array}{l}\text { Alter, Geschlecht, Gewicht, } \\
\text { Größe, Körperoberfläche }\end{array}$ \\
$\begin{array}{l}\text { Aktuelle Medikation } \\
\text { Erwartungswerte }\end{array}$ & Leistung, Herzfrequenz \\
Art der Belastung & Laufband-, Fahrradergometer, \\
Ruhe-EKG-Befund & Wattstufengröße, Stufendauer \\
Name des Untersuchers & \\
Datum, Uhrzeit & \\
\hline Während und nach der Belastung \\
\hline Grafische Dokumentation & $\begin{array}{l}\text { Herzfrequenz, Blutdruck, EKG- } \\
\text { Auffälligkeiten }\end{array}$ \\
Subjektive Symptome & PER, AP, andere (s. Tabellen \\
PER, AP, andere) \\
$\begin{array}{l}\text { Abbruchkriterium } \\
\text { Leistungsfähigkeit }\end{array}$ \\
$\begin{array}{l}\text { Absolut (Watt bzw. km/h und } \\
\text { Steigung, MET) } \\
\text { Relativ (\% Soll), W/kg) } \\
\text { Oualitative und semiquantita- } \\
\text { tive Beurteilung von Rhythmus- } \\
\text { störungen und ST-Strecke }\end{array}$ \\
\hline
\end{tabular}

(84\%) ergibt. Allerdings verringert sich dadurch die Spezifität $(70 \%)$ - bedingt durch häufig vorbestehende Veränderungen des Ruhe-EKGs (frühere durchgemachte Myokardinfarkte, intraventrikuläre Herzreizleitungsstörungen) und/oder einer Linkshypertrophie [101, 102].

\subsection{Komorbidität}

Ein Problem bei der Interpretation der Untersuchung kann eine fehlende Ausbelastung aufgrund diverser Begleiterkrankungen sein.

Weitere Einflussgrößen auf das Ergometrieergebnis im Sinne von falsch positiven Befunden und falsch negativen Befunden sind in den Tabellen 20 und 21 dargestellt.

\section{Dokumentation}

Die gesamte Belastungsuntersuchung bedarf einer standardisiert protokollierten Dokumentation. In dieser Dokumentation sollten die aus Tabelle 22 ersichtlichen Punkte enthalten sein.

Ein Beispiel einer Protokollierung der Ergometrie ist im Anhang dargestellt.

\section{Literatur:}

1. Böhm H, Bürklen R, Dienstl F, Ehrenböck R, Gaul G, Herbinger W, Kiss E, Kubicek F, Kühn P, Kummer F, Niederberger M, Schlick W. Empfehlungen für eine standardisierte Ergometrie. Öst Ärzteztg 1978; 33: 333-44.

2. Stuart RJ, Ellestad MH. National survey of exercise testing. Chest 1980; 77: 94-7.

3. Niederberger M, Böhm H, Ehrenböck $R$, Gasic S, Gaul G, Kiss E, Kubicek F, Sudhas P. Methodische Begründung für den ergometrischen Standardisierungsvorschlag. Öst Ärzteztg 1978; 33: 345-51.

4. Gibbons RJ, Balady GJ, Bricker T, Chaitman BR, Fletcher GF, Froelicher VF, Mark DB McCallister BD, Mooss AN, O'Reilly MG, Winters WL. ACC/AHA 2002 Guideline Update for Exercise Testing. A Report of the American College of Cardiology/American Heart Association. Task Force on Practice Guidelines (Committee on Exercise Testing). Circulation 2002; 106: 1883-92.

5. Fletcher GF, Balady GJ, Amsterdam EA, Chaitman B, Eckel R, Fleg J, Froelicher VF, Leon AS, Piña IL, Rodney R, Simons-Morton DA, Williams MA, Bazzarre T. Exercise Standards for Testing and Training: A Statement for Healthcare Professionals From the American Heart Association. Circulation 2001; 104: 1694-740

6. Trappe HJ, Löllgen H. Leitlinien zur Ergometrie. Z Kardiol 2000; 89: 821-37.

7. Gersh BJ, Braunwald E, Rutherford JD. Chronic coronary artery disease. In: Braunwald E (ed). Heart Disease. $5^{\text {th }}$ ed. WB Saunders Company, Philadelphia, 1997; 1289-365.

8. Pokan R, Hofmann P, Von Duvillard SP Beaufort F, Schumacher M, Fruhwald FM, Zweiker R, Eber B, Gasser R, Brandt D, Smekal G, Klein W, Schmid P. Left ventricular function in response to the transition from aerobic to anaerobic metabolism. Med Sci Sports Exerc 1997: 29: 1040-7.

9 . Skinner JS, McLellan TH. The Transition from Aerobic to Anaerobic Metabolism. Res 0 Exerc Sport 1980; 51: 234-48.
10. Pokan R, Hofmann P, Wonisch M. Dreiphasigkeit der Energiebereitstellung. In: Pokan R, Förster H, Hofmann P, Hörtnagl H, Ledl-Kurkowski E, Wonisch M (Hrsg). Kompendium der Sportmedizin. Physiologie, Innere Medizin und Pädiatrie. Springer, BerlinHeidelberg-New York, 2004; 39-44.

11. Hofmann P, Wonisch M, Pokan R. Laktatleistungsdiagnostik. Durchführung und Interpretation. In: Pokan R, Förster H, Hofmann $P$, Hörtnagl $H$, Ledl-Kurkowski E, Wonisch M (Hrsg). Kompendium der Sportmedizin. Physiologie, Innere Medizin und Pädiatrie. Springer, Berlin-Heidelberg-New York, 2004; 103-132.

12. Wonisch M, Pokan R, Hofmann P. Funktionsdiagnostik akuter und chronischer Anpassung der Atmungsorgane. In: Pokan R, Förster H, Hofmann P, Hörtnagl H, LedlKurkowski E, Wonisch M (Hrsg). Kompendium der Sportmedizin. Physiologie, Innere Medizin und Pädiatrie. Springer, Berlin-Heidelberg-New York, 2004; 133-144.

13. Hofmann P, Pokan R, Preidler K, Leitner $H$ Szolar D, Eber B, Schwaberger G. Relationship between heart rate threshold, lactate turn point and myocardial infarction. Int J Sports Med 1994; 15: 232-7.

14. Pokan R, Hofmann P, Von Duvillard SP, Smekal G, Gasser R, Eber B, Klein W, Bachl N. Schmid P. The heart rate performance curve and left ventricular function during exercise in patients after myocardial infarction. Med Sci Sports Exerc 1998; 30: 147580.

15. Hofmann P, Seibert F-J, Zweiker B Schmid $P$. The heart rate performance curve during incremental cycle ergometer exercise in healthy young male subjects. Med Sci Sports Exerc 1997; 29: 762-8.

16. Foster C, Porcari JP. Clinical exercise testing related to cardiovascular disease. In: Kaminsky LA (ed). ACSM's Resource Manual for Guidelines for Exercise testing and Prescription. $5^{\text {th }}$ ed. Lippincott Williams \& Wilkins, Philadelphia, 2006; 225-30.

17. ACC/AHA guidelines for exercise testing: a report of the American College of Cardiol- 
ogy/American Heart Association Task Force on Practice Guidelines Committee on Exercise Testing. J Am Coll Cardiol 1997; 30: 260315

18. Zipes DP, Camm J, Borggrefe M, Buxton AE, Chaitman B, Fromer M, Gregoratos G, Klein G, Moss AJ, Myerburg RJ, Priori SG, Quinones MA, Roden DM, Silka MJ, Tracy C. ACC/AHA/ESC 2006 guidelines for management of patients with ventricular arrhythmias and the prevention of sudden cardiac death executive summary. A report of the America College of Cardiology/American Heart Association Task Force and the European Society of Cardiology Committee for Practice Guidelines (Writing Committee to Develop Guidelines for Management of Patients with Ventricular Arrhythmias and the Prevention of Sudden Cardiac Death) Developed in collaboration with the European Heart Rhythm Association and the Heart Rhythm Society. Eur Heart J 2006; 27: 2099-140.

19. Fuster V, Rydén LE, Cannom DS, Crijns HJ, Curtis AB, Ellenbogen KA, Halperin JL, Heuzey J-YL, Kay GN, Lowe JE, OIsson SO, Prystowsky EN, Tamargo JL, Wann S. ACC/ AHA/ESC 2006 guidelines for the management of patients with atrial fibrillation - executive summary. A report of the American College of Cardiology/American Heart Association Task Force on practice guidelines an the European Society of Cardiology Committee for Practice Guidelines (Writing Committee to Revise the 2001 Guidelines for the Management of Patients with Atrial Fibrilla tion). Developed in collaboration with the European Heart Rhythm Association and the Heart Rhythm Society. Eur Heart J 2006; 27 : 1979-2030.

20. Wonisch $\mathrm{M}$, Lercher P. Scherr D, Maier $\mathrm{B}$ Pokan R, Hofmann P, Von Duvillard SP. Influence of Permanent Right Ventricular Pacing on Cardiorespiratory Exercise Parameters in Chronic Heart Failure Patients with Implanted Cardioverter Defibrillators. Chest 2005; 127 : 787-93.

21. Slany J, Magometschnigg D, Mayer G, Pichler M, Pilz H, Rieder A, Schernthaner G, Skrabal F, Silberbauer K, Stoschitzky K Watschinger B, Zweiker R. Klassifikation, Diagnostik und Therapie der Hypertonie 2007 - Empfehlungen der Österreichischen Gesellschaft für Hypertensiologie. J Hypertonie 2007; 11: 7-11.

22. Mancia G, De Backer G, Dominiczak A, Cifkova R, Fagard R, Germano G, Grassi G, Heagerty AM, Kjeldsen SE, Laurent S, Narkiewicz K, Ruilope L, Rynkiewicz A Schmieder RE, Struijker Boudier HAJ, Zanchetti A. 2007 Guidelines for the Management of Arterial Hypertension - The Tas Force for the Management of Arterial Hypertension of the European Society of Hypertension (ESH) and of the European Society of Cardiology (ESC). J Hypertens 2007; 25: 1105-87.

23. Franz IW. Blood pressure measurement during ergometric stress testing. Z Kardiol 1996; 85 (Suppl 3); 71-5.

24. Vahanian $A$, Baumgartner $H, B a x ~ J$, Butchart E, Dion R, Filippatos G, Flachskamp F, Hall R, Lung B, Kasprzak J, Nataf P, Torno $P$, Torracca L, Wenink A. Guidelines on the management of valvular heart disease. The task Force on the Management of Valvular Heart Disease of the European Society of Cardiology. Eur Heart J 2007; 28: 230-68.

25. Bonow RO, Carabello BA, Chatterjee K, de Leon AC Jr, Faxon DP, Freed MD, Gaasch WH, Lytle BW, Nishimura RA, O'Gara PT, O'Rourke RA, Otto CM, Shah PM, Shanewis JS, Smith SC Jr, Jacobs AK, Adams CD, Anderson JL, Antman EM, Faxon DP, Fuster V, Halperin JL, Hiratzka LF, Hunt SA, Lytle BW Nishimura R, Page RL, Riegel B. ACC/AHA 2006 Practice Guidelines for the Management of Patients With Valvular Heart Dis- ease: Executive Summary: A Report of the American College of Cardiology/American Heart Association Task Force on Practice Guidelines (Writing Committee to Revise the 1998 Guidelines for the Management of Patients With Valvular Heart Disease) Developed in Collaboration With the Society of Cardiovascular Anesthesiologists Endorsed by the Society for Cardiovascular Angiography and Interventions and the Society of Thoracic Surgeons. J Am Coll Cardiol 2006; 48 . 598-675.

26. Maron BJ, McKenna WJ, Danielson GK, Kappenberger LJ, Kuhn HJ, Seidman CE, Shah PM, Spencer WH, Spirito P, Ten Cate FJ, Wigle ED. American College of Cardiology/European Society of Cardiology Clinical Expert Consensus Document on Hypertrophic Cardiomyopathy. A report of the American College of Cardiology Foundation Task Force on Clinical Expert Consensus Documents and the European Society of Cardiology Committee for Practice Guidelines. Eur Heart J 2003; 24: 1965-91.

27. ESC. Working Group on cardiac Rehabilitation \& exercise Physiology and working Group on Heart Failure of the European Society of Cardiology. Recommendations for exercise testing in chronic heart failure patients. Eur Heart J 2001; 22: 37-45.

28. ACSM's Guidelines for exercise testing and prescription. $6^{\text {th }}$ ed. Lippincott Williams \& Wilkins, Philadelphia, 2006; 91-114.

29. Buchfuhrer MJ, Hansen JE, Robinson TE, Sue DY, Wasserman K, Whipp BJ. Optimizing the exercise protocol for cardiopulmonary assessment. J Appl Physiol 1983; 55: 155864.

30. Hofmann P, Pokan R. Belastungsuntersuchungen und Protokolle. In: Pokan R, Benzer W, Gabriel H, Kunschitz E, Mayr K, Sarnitz G,
Schindler K (Hrsg). Kompendium der Kardiologischen Prävention und Rehabilitation. Springer-Verlag, Wien, 2008 (in press).

31. Lauer MS, Francis GS, Okin PM, Pashkow FJ, Snader CE, Marwick TH. Impaired chronotropic response to exercise stress testing as a predictor of mortality. JAMA 1999; 281: 524-9.

32. Heck H, Rost R, Hollmann W. Normwerte des Blutdrucks bei der Fahrradergometrie. Deutsch Z Sportmed 1984; 35: 243-7.

33. Singh JP, Larson MG, Manolio TA, O'Donnell CJ, Lauer M, Evans JC, Levy D. Blood pressure response during treadmill testing as a risk factor for new-onset hypertension: the Framingham heart study. Circulation 1999; 99: 1831-6.

34. Miyai N, Arita H, Miyashita K, Morioka I, Shiraishi T, Nishio I. Blood pressure response to heart rate during exercise test and risk of future hypertension. Hypertension 2002; 39: 761-6.

35. American College of Sports Medicine: Po sition stand: Exercise and hypertension Med Sci Sports Exerc 2004; 36: 533-53.

36. Lim PO, MacFadyen RJ, Clarkson PB. Impaired exercise tolerance in hypertensive patients. Ann Intern Med 1996; 124: 41-55.

37. Otterstad JE, Davies M, Ball SG Left ventricular hypertrophy and myocardial ischaemia in hypertension: the THAMES Study. Eur Heart J 1993; 14: 1622-8.

38. Laukanen JA, Kurl S, Rauramaa R, Lakka TA, Venälainen JM, Salonen JT. Systolic blood pressure response to exercise testing is related to the risk of acute myocardial infarction in middle-aged men. Eur $\mathrm{J}$ Cardiovasc Prev Rehabil 2006; 13: 421-8.

39. McHam SA, Marwick TH, Paskow FJ, Lauer MS. Delayed systolic blood pressure recovery after graded exercise: An independent correlate of angiographic coronary disease. J Am Coll Cardiol 1999; 34: 754-9.
40. Frolkis JP, Pothier CE, Blackstone EH, Lauer MS. Frequent ventricular ectopy after exercise as a predictor of death. N Engl J Med 2003; 348: 781-90.

41. Dewey FE, Kapoor JR, Williams RS, Lipinski MJ, Aschley EA, Hadley D, Myers J, Froelicher VF. Ventricular arrhythmias during clinical treadmill testing and prognosis. Arch Intern Med 2008; 168: 225-34.

42. Tegtbur U, Meyer H, Machold H, Busse MW. Belastungsdiagnostische Kenngrößen und Katecholamine bei Koronarpatienten. Z Kardiol 2002: 91: 927-36.

43. Wonisch M, Hofmann P, Schmid P, Pokan R. Zusammenhang zwischen anaerober Schwelle, Katecholaminen und Arrhythmien bei Patienten mit Herzerkrankungen. Österr J Sportmed 2007; 37: 50-6.

44. Pokan R, Hofmann P, Smekal G, Wonisch M, Bachl N, Schmid P. Leistungsdiagnostik und Trainingsherzfrequenzbestimmung in de kardiologischen Rehabilitation. Leistungsdiagnostik zur Trainingssteuerung in der Bewegungstherapie von Herz-Kreislauferkrankungen. Internist Prax 2002; 42: 797-806.

45. Pokan R, Von Duvillard SP, Ludwig J, Rohrer A, Hofmann P, Wonisch M, Smekal G, Schmid P Pacher R, Bachl N. Effect of high. volume and intensity endurance training in heart transplant recipients. Med Sci Sports Exerc 2004; 36: 2011-6.

46. Wonisch M, Hofmann P, Fruhwald FM, Kraxner W, Hödl R, Pokan R, Klein W. Influence of beta-blocker use on percentage of target heart rate exercise prescription. Eur $J$ Cardiovasc Prev Rehabil 2003; 10: 296-301.

47. Pokan R, Hofmann P, Wonisch M, Smeka G, Bachl N, Mayr K, Benzer W, Schmid P. Leistungsdiagnostik und Trainingsherzfrequenzbestimmung in der kardiologischen Rehabilitation. J Kardiol 2004; 11: 446-52.

48. Binder RK, Wonisch M, Corra U, CohenSolal A, Vanhees L, Saner H, Schmid J-P. Methodological approach to the $1^{\text {st }}$ and $2^{\text {nd }}$ lactate threshold in incremental cardiopulmonary exercise testing. Eur J Cardiovasc Prev Rehab 2008; (in press).

49. Pokan R, Hofmann P, Von Duvillard SP, Smekal G, Wonisch M, Lettner K, Schmid P, Shechter M, Silver B, Bachl N. Oral magnesium therapy, exercise heart rate, exercise tolerance, and myocardial function in coronary artery disease patients. Br J Sports Med 2006; 40: 773-8.

50. Wonisch M, Hofmann P. Fruhwald FM, Höd R, Schwaberger G, Pokan R, Von Duvillard SP Klein W. Effect of $\beta 1$-selective adrenergic blockade on maximal lactate steady state in healthy men. Eur J Appl Physiol 2002; 87 : 66-71.

51. Hofmann P, Bunc V, Leitner H, Pokan R, Gaisl R. Heart rate threshold related to lactate turn point and steady state exercise on cycle ergometer. Eur J Appl Physiol 1994; 69 132-9.

52. Urhausen A, Kindermann W. Trainingsempfehlungen im Gesundheitssport. In:

Kindermann W, Dickhuth HH, Niess A, Röcke K, Uhrhausen A (Hrsg). Steinkopf-Verlag, Darmstadt, 2003; 35-50.

53. Hofmann P, Wonisch M, Pokan R, Schwaberger G, Smekal G, Klein W, Von Duvillard SP. $\beta 1$-adrenoceptor mediated origin of the heart rate performance curve deflection. Me Sci Sports Exerc 2005; 37: 1704-9.

54. Hofmann P, Von Duvillard SP, Seibert FJ, Pokan R, Wonisch M, LeMura LM, Schwaberger G. \%HRmax target heart rate is dependent on heart rate performance curve deflection. Med Sci Sports Exerc 2001; 10: 172631

55. Karvonen MJ, Kental E, Mustal 0. The effects of Training on Heart Rate Ann Med Exp et Biol Fenn 1957; 35: 307

56. Stuart RJ, Ellestad MH. National survey of exercise testing. Chest 1980; 77: 94-7.

57. Hamm LF, Crow RS, Stull GA, Hannan P. Safety and characteristics of exercise testing early after acute myocardial infarction. Amer J Cardiol 1989; 63: 1193-7.

58. Juneau M, Colles $P$, Théroux $P$, de Guise $P$, Pelletier G, Lam J, Waters D. Symptom-limited versus low level exercise testing befor hospital discharge after myocardial infarction. J Am Coll Cardiol 1992; 20: 927-33.

59. Jain A, Myers GH, Sapin PM, O'Rourke RA. Comparison of symptom-limited and low level exercise tolerance tests early after myocardial infarction. J Am Coll Cardiol 1993; 22: 1816-20.

60. Mittleman MA, Maclure M, Tofler GH, Sherwood JB, Goldberg RJ, Muller JE. Triggering of acute myocardial infarction by heavy physical exertion: protection against triggering by regular exertion: Determinants of Myocardial Infarction Onset Study Investigators. N Engl J Med 1993; 329: 1677-83.

61. Willich SN, Lewis M, Löwel H, Arntz HR, Schubert F, Schröder R. Physical exertion as a trigger of acute myocardial infarction: Triggers and Mechanisms of Myocardial Infarction Study Group. N Engl J Med 1993; 329: 1684-90

62. Tofler GH, Muller JE, Stone PH, Forman S, Solomon RE, Knatterud GL, Braunwald E.

Modifiers of timing and possible triggers of acute myocardial infarction in the Thrombolysis in Myocardial Infarction Phase II (TIMI II) Study Group. J Am Coll Cardiol 1992; 20: 1049-55. 
tor of death. N Engl J Med 2003: 348: $2357-$

73. Young DZ, Lampert S, Graboys TB, Lown B. Safety of maximal exercise testing in patients at high risk for ventricular arrhythmia. Circulation 1984; 70: 184-91.

74. Saini V, Graboys TB, Towne V, Lown B. Reproducibility of exercise-induced ventricular arrhythmia in patients undergoing evaluation for malignant ventricular arrhythmia. Am J Cardiol 1989; 63: 697-70

75. Jezior MR, Kent SM, Atwood JE. Exercise Testing in Wolff- Parkinson-White Syndrome - Case report with ECG and literature review. Chest 2005; 127: 1454-7.

76. Corbelli R, Masterson M, Wilkoff BL. Chronotropic response to exercise in patients with atrial fibrillation. Pacing Clin Electrophysiol 1990; 13: 179-87.

77. Pinski SL, Fahy GJ. The proarrhythmic potential of implantable cardioverter-defibrillators. Circulation 1995; 92: 1651-64.

78. Ellestad MH, Wan MK. Predictive implications of stress testing: follow-up of 2700 subjects after maximum treadmill stress testing. Circulation 1975; 51: 363-9.

79. Lauer MS, Okin PM, Larson MG, Evans JC, Levy D. Impaired heart rate response to graded exercise. Prognostic implications of chronotropic incompetence in the Framingham Heart Study. Circulation 1996; 93: 15206.

80. Wilkoff BL, Miller RE. Exercise testing for chronotropic assessment. Cardiology Clinics 1992; 10: 705-17.
81. Lauer MS, Francis GS, Okin PM, Pashkow FJ, Snader CE, Marwick TH. Impaired chronotropic response to exercise stress testing as a predictor of mortality. JAMA 1999; 281: 524-9.

82. Lauer MS, Mehta R, Pashkow FJ, Okin PM, Lee K, Marwick TH. Association of chronotropic incompetence with echocardiographic ischemia and prognosis. J Am Coll Cardiol 1998; 32: 1280-6.

83. Azarbal B, Hayes SW, Lewin HC, Hachamovitch R, Cohen I, Berman DS. The incremental prognostic value of percentage of heart rate reserve achieved over myocardial perfusion SPECT in the prediction of cardiac death and all-cause mortality: superiority over $85 \%$ of age-predicted maximum heart rate. J Am Coll Cardiol 2004; 44: 423 30.

84. Lauer M, Froelicher ES, Williams M, Kligfield P. Exercise Testing in Asymptomatic Adults: A Statement for Professionals From the American Heart Association Council on Clinical Cardiology Subcommittee on Exercise, Cardiac Rehabilitation, and Prevention. Circulation 2005; 112: 771-6.

85. Lele SS, Scalia G, Thomson H, Macfarlane D. Wilkinson D Stafford W, Khafagi F,

Frenneaux M. Mechanism of exercise hypotension in patients with ischemic heart disease. Role of neurocardiogenically mediated vasodilation. Circulation 1994; 90: 2701-9.

86. Rehman A, Zelos G, Andrews NP, Mulcahy D, Quyyumi AA. Blood pressure changes during transient myocardial ischemia: Insights into mechanisms. J Am Coll Cardiol 1997; 30 1249-55.
87. Dubach P, Froelicher VF, Klein J, Oakes D, Grover-McKay M. Friis R. Exercise-induced hypotension in a male population: criteria causes, and prognosis. Circulation 1988; 78 $1380-7$

88. Watson G, Mechling E, Ewy GA. Clinical significance of early vs late hypotensive blood pressure response to treadmill exercise. Arch Intern Med 1992; 152: 1005-8.

89. Ellestad MH, Stuart RJ. Exercise stres testing: principles and clinical application. In: Parmley WW, Chatterjee K (eds). Cardiology. JB Lippincott Company, Philadelphia, 1992; Chapter 45

90. Derman WE, Sims R, Noakes TD. The effects of antihypertensive medications on the physiological response to maximal exercise testing J Cardiovasc Pharmacol 1992: 19: 122-7.

91. Richardson RS, Leek BT, Wagner PD, Kritchevsky M. Transient global amnesia: a complication of incremental exercise testing Med Sci Sports Exerc 1998; 30 (10 Suppl): 403-5.

92. American College of Cardiology. 32 Bethesda Conference: Care of the adult with congenital heart disease. J Am Coll Cardiol 2001; 37: 1161-98.

93. Grady TA, Chiu AC, Snader CE, Marwick TH, Thomas JD, Pashkow FJ, Lauer MS. Prognostic significance of exercise-induced left bundle-brunch block. JAMA 1998; 279: 153-

94. Bunch TJ, Chandrasekaran K, Gersh BJ, Hammill SC, Hodge DO, Khan AH, Packer DL, Pellikka PA. The prognostic significance of exercise-induced atrial arrhythmias. J Am Coll Cardiol 2004; 43: 1236-40. 95. Jelinek MV, Lown B. Exercise stress testing for exposure of cardiac arrhythmia. Prog Cradiovasc Dis 1974; 16: 497-522.

96. Curzen N. Patel D, Clarke D, Wright C Mulcahy D, Sullivan A, Holdright D, Rox K. Women with chest pain: Is exercise testing worthwhile? Heart 1996; 76: 156-60.

77. D'Antono B, Dupuis G, Fortin C, Arsenault A. Burelle D. Angina symptoms in men and women with stable coronary artery disease and evidence of exercise-induced myocardial perfusion defects. Am Heart J 2006; 151 813-90.

98. Sketch M, Mohiuddin S, Lynch J, Zencka $A E$, Runco V. Significant sex differences in the correlation of electrocardiographic exercise testing and coronary angiography. Am J Cardiol 1975; 36: 169-73.

99. Sketch MH, Mooss AN, Butler MN Digoxin-induced positive exercise tests: their clinical and prognostic significance. Am J Cardiol 1981; 48: 655-9.

100. Anastasion-Nana MI, Anderson JL, Stewart JR, Crevey BJ. Occurrence of exercise induced and Spontaneous wide complex tachycardia during therapy with flecainide for complex ventricular arrhythmias: a probable pro arrhythmic effect. Am Heart J 1987; 113: 1071-7.

101. Hlatky MA, Pryor DB, Harrell FE Jr. Factors effecting sensitivity and specificity of exercise electrocardiagraphy: multivariable analysis. Am J Med 1984; 77: 64-71. 102. Goraya TY, Jacobsen SJ, Pellikka PA. Prognostic value of treadmill exercise testing in elderly persons. Ann Intern Med 2000; 132: 862-70. 


\section{Anhang - Untersuchungsprotokoll}

STEMPEL

Protokoll und Befund

Standardisiertes Untersuchungsblatt der

Österr. Kardiol. Gesellschaft

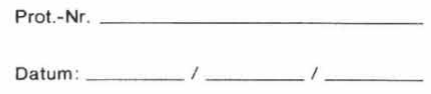

Tag / Monat / Jahr

Name:

Alter

Zugewiesen:

GröBe: Gewicht: Körperoberfläche

Fragestellung:

Eingenommene Medikamente:

Ruhe-12-Abl. EKG:

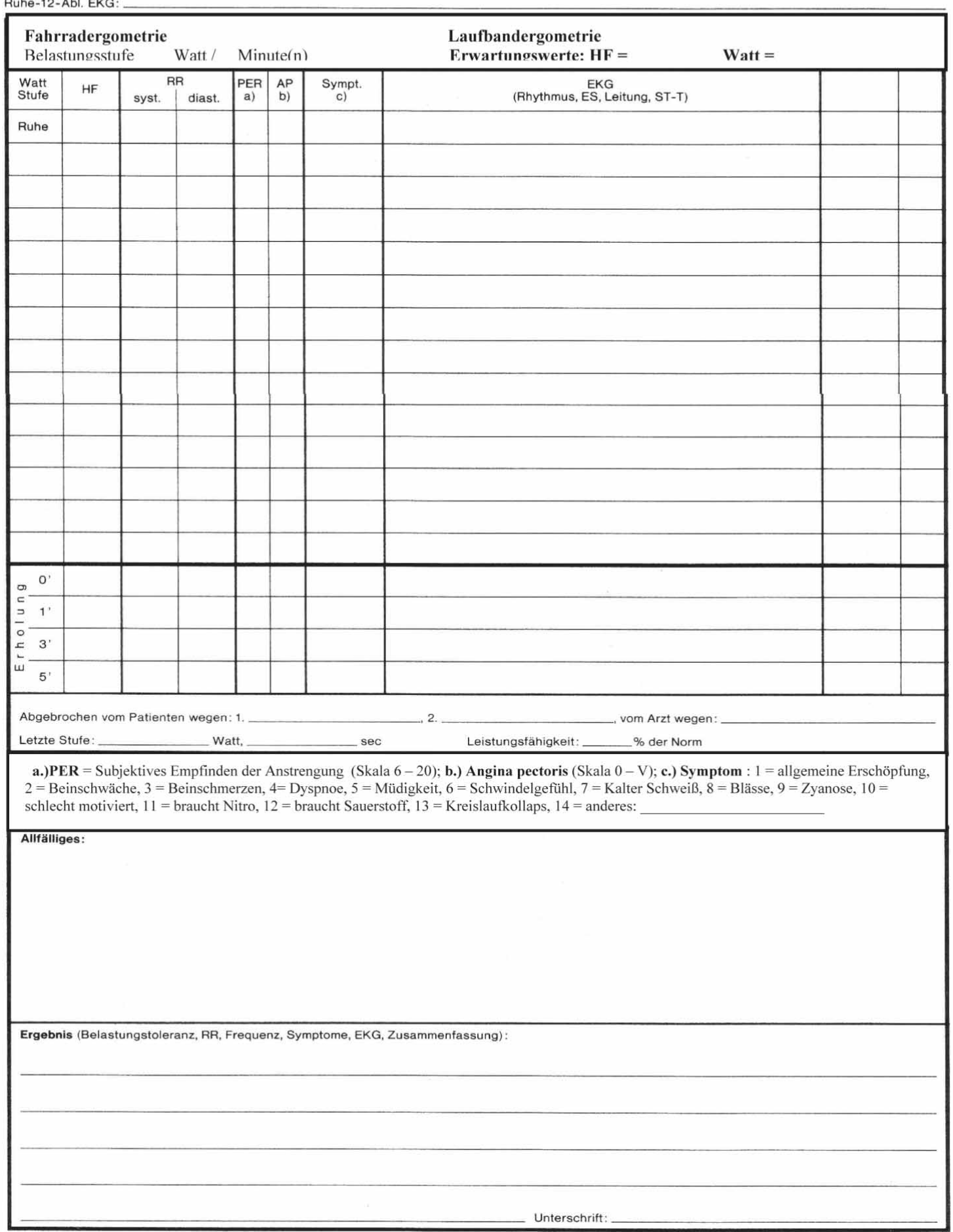




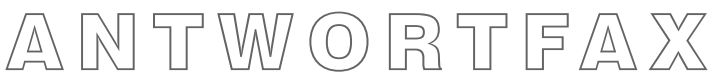

\section{JOURNAL FÜR KARDIOLOGIE}

\author{
Hiermit bestelle ich \\ ein Jahresabonnement \\ (mindestens 6 Ausgaben) zum \\ Preis von $€ 60,-$ (Stand 1.1.2009) \\ (im Ausland zzgl. Versandspesen)
}

Name

Datum, Unterschrift

\section{Einsenden oder per Fax an:}

Krause \& Pachernegg GmbH, Verlag für Medizin und Wirtschaft, Postfach 21, A-3003 Gablitz, FAX: +43 (0) 2231 / 612 58-10 